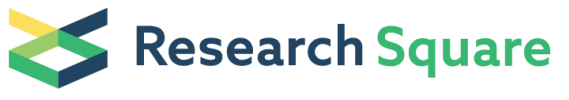 \\ Preprints are preliminary reports that have not undergone peer review. \\ They should not be considered conclusive, used to inform clinical practice, \\ or referenced by the media as validated information.
}

\section{Speckle-Tracking Echocardiography is Preferable Measurement for Detecting the Early and Subtle Alterations of Cardiac Dysfunction in Diabetic Rats}

\author{
Zhiyan Chen \\ Hebei Medical University \\ Yanchao Qi \\ Hebei Medical University First Affiliated Hospital \\ Lijie Wang \\ Hebei Medical University No2 Hospital: Second Hospital of Hebei Medical University \\ Meifang Ma \\ Hebei Medical University No2 Hospital: Second Hospital of Hebei Medical University \\ $\mathrm{Na} \mathrm{Li}$ \\ Hebei Medical University No2 Hospital: Second Hospital of Hebei Medical University \\ Zhe Liu \\ Hebei Medical University No2 Hospital: Second Hospital of Hebei Medical University \\ Suyun Liu \\ Hebei Medical University No2 Hospital: Second Hospital of Hebei Medical University \\ Lixiang Xue \\ Peking University Third Hospital \\ Bingyan Guo \\ Hebei Medical University No2 Hospital: Second Hospital of Hebei Medical University \\ Yongjun Li ( $\sim$ lyjbs2009@163.com ) \\ Hebei Medical University No2 Hospital: Second Hospital of Hebei Medical University
}

https://orcid.org/0000-0002-5103-7861

\section{Original investigation}

Keywords: Diabetic cardiomyopathy, Myocardial damage, inhibiting RhoA/ROCK signaling pathway, cardiac function, Speckle-tracking echocardiography

Posted Date: April 1st, 2021

DOI: https://doi.org/10.21203/rs.3.rs-371725/v1

License: (c) (i) This work is licensed under a Creative Commons Attribution 4.0 International License. Read Full License 


\section{Abstract}

\section{Background}

Myocardial damage caused by diabetic cardiomyopathy results in cardiac structural and functional abnormalities. Our previous studies have demonstrated that inhibiting RhoA/ROCK signaling pathway improved the structural abnormalities. The early detection of cardiac functional alterations accompanied by structural changes during therapy may facilitate better understanding on the pathophysiologic progress and guiding diabetic cardiomyopathy treatment. This study aimed to identify the optimal diagnostic measures for the early and subtle alterations of cardiac dysfunction in Type 2 diabetes mellitus rats.

Methods

Twenty-four male Wistar rats were randomly divided into four groups and treated for 4 weeks: CON (control rats), DM (diabetic rats), DMF (DM + fasudil $10 \mathrm{mg} / \mathrm{kg} / \mathrm{d}$ ) and CONF (CON + fasudil 10mg/kg/d). Left ventricular (LV) structure was quantified by histological staining and transmission electron microscopy. LV function and myocardial deformation were performed by high-frequency echocardiography.

Results

Myocardial hypertrophy and fibrosis were increased in diabetic rats and were remarkably alleviated in the DMF group. The diabetic rats had impaired LV performance evidenced by significant reduction of EF, FS and MV E/A, 26\%, 34\% and 20\% respectively, while ROCK inhibition failed to improve the conventional ultrasonic parameters. However, the cardiac time intervals (CTI) parameters and speckle-tracking echocardiography (STE) parameters were significantly improved in the DMF group compared with the DM group (isovolumic contraction time, IVCT: $P=0.029$; myocardial performance index, MPI: $P=0.037$; fractional area change, FAC: $P<0.001$; global circumferential strain, GCS: $P=0.003$; global circumferential strain rate, GCSR: $P=0.021)$. Combining ROC curves with linear regression analysis, STE parameters were characterized by both the optimal predictions for cardiac damage [AUC ( $95 \% \mathrm{Cl})$ : FAC, 0.927 ( 0.744 to 0.993$)$; GCS, 0.819 (0.610 to 0.945); GCSR, 0.899 (0.707 to 0.984)] and the strong correlations with cardiac fibrosis (FAC, $r=-0.825 ; G C S, r=0.772 ; G C S R, r=0.829)$.

\section{Conclusion}

The results suggest that STE strain and strain rate are preferable indicators of the early detection of subtle alterations in cardiac dysfunction and the quantitation of therapeutic efficacy in diabetic cardiomyopathy.

\section{Background}

Diabetes mellitus (DM) remains a global prevalence with more than 450 million adult patients worldwide, and the number is expected to reach 693 million until 2045[1]. Diabetic cardiomyopathy, acting as a serious cardiovascular complication induced by DM in particular type 2 diabetes mellitus (T2DM)[2], contributes to the incidence of the composite endpoint of worsening of heart failure (HF) or death. The term diabetic cardiomyopathy is defined as a series of structural abnormalities, including a diffuse myocardial fibrosis, cardiomyocyte hypertrophy and coronary microvascular disorder, and cardiac dysfunction independent of the cardiovascular risk factors, such as hypertension, atherosclerosis or coronary artery disease $[3,4]$.

With a growing understanding of the underlying mechanisms, RhoA/ROCK signaling pathway is proposed to contribute to the pathogenesis of diabetic cardiomyopathy[5-7]. Our previous studies have shown that inhibiting RhoA/ ROCK signaling pathway improved the structural abnormalities in diabetic hearts[8-10]. The changes of cardiac structure are usually accompanied with functional alterations. However, we have not observed the cardiac functional improvements in diabetic animals by traditional ultrasonic parameters. The early detection of cardiac performance during therapy may facilitate better understanding on the pathophysiologic progress and guiding diabetic cardiomyopathy treatment. Thus, there is an urgent need for diagnostic approaches for the early identification of cardiac functional alterations to predict diabetic cardiomyopathic process and therapeutic effect.

Transthoracic echocardiography is a standard non-invasive method of cardiac function assessment both in animal models and humans[11, 12]. Conventional parameters, such as EF and FS, are widely used as surrogate markers of cardiac function[13]. However, the modest sensitivity and technical limitations make them fail to analyze the subtle changes of myocardial damage. Speckle-tracking echocardiography (STE) is a highly sensitive technique used for characterizing myocardium deformation by global and segmental strain parameters[14-16]. Previous studies have suggested that myocardial strain and strain rate are capable of detecting the subtle differences of pressure overload induced myocardial remodeling between young and adult rats[14]. Most notably, longitudinal strain (LS) parameters are well-accepted surrogates of putative subendocardial damage and may serve as diagnostic markers of microstructural remodeling[15]. Moreover, parameters derived from STE are valuable to describe the LV dynamic changes in active relaxation and contractility in T1DM and 
T2DM animal models[17]. Whereas, whether STE can be a sensitive diagnostic approach of diabetic cardiomyopathy to evaluate the subtle cardiac functional alterations and predict the therapeutic efficacy in the early stage is still lack of evidences.

In the present study, we aimed to investigate the subtle changes in cardiac structure and function in T2DM induced diabetic cardiomyopathy rat models after treated with a Rho-kinase inhibitor. By combining comprehensive STE analyses with several other measures, we sought to provide translational evidence for diabetic cardiomyopathy management. We tested the hypothesis that the early protective actions of ROCK inhibition on cardiac remodeling is detectable by assessing the subtle functional alterations by STE in diabetic cardiomyopathy rats.

\section{Methods}

\section{Animals}

Experimental protocols used in this study followed the guidelines for the Care and Use of Laboratory Animals published by the US National Institutes of Health (1996) and were approved by the research ethic committee of Hebei Medical University. Male Wistar rats (Rattus norvegicus), 4 weeks old, were obtained from the Vital River Laboratory (Beijing, China). All rats were housed in a temperature-controlled room on a $12 \mathrm{~h} \mathrm{light/dark} \mathrm{cycle} \mathrm{with} \mathrm{free} \mathrm{access} \mathrm{to} \mathrm{water} \mathrm{and} \mathrm{standard} \mathrm{rodent} \mathrm{chow.}$

Following 1 week of acclimatization, the rats were randomly divided into two groups: control and diabetes. After 6 weeks of high-fat diet (HFD, 60 kcal\% fat, D12492, Vital River Laboratory, Beijing, China) feeding, the rats were fasted for $8 \mathrm{~h}$, and then were intraperitoneally injected with a single dose of Streptozocin (STZ, $35 \mathrm{mg} / \mathrm{kg}$; S0130, sigma, USA) dissolved in $0.1 \mathrm{M}$ citrate buffer (pH 4.5) to induce T2DM as previously described[18]. As controls, the rats received a single intraperitoneal injection of citrate buffer alone and were fed with normal diet as before. After 3 days, 1 weeks and 4 weeks of STZ-injection, fast blood glucose (FBG) level was measured by blood glucose meter (yuwell580, China) using tail vein blood sample. FBG $>11.1 \mathrm{mmol} / \mathrm{I}$ was considered as diabetes. All the diabetic rats were kept on HFD feeding for 24 weeks.

\section{Experimental design}

After 24 weeks for diabetes, the normal and diabetic rats, respectively and randomly, were divided into 4 groups (at least 6 rats in each group): the CON (control rats) group, the DM (diabetic rats) group, the DMF (diabetic rats + fasudil) group and the CONF (control rats + fasudil) group. The rats from the DMF group and CONF group were treated with fasudil ( $10 \mathrm{mg} / \mathrm{kg} / \mathrm{d}$; Hongri, Tianjin, China) by intraperitoneal injection. The CON group and DM group were intervened with an equal volume of saline intraperitoneally per day. After 4-week treatment, rats were sacrificed for the collections of heart tissues for further analysis (Fig.S1).

\section{Histological analysis}

LV samples of all rats were harvested and fixed with $4 \%$ paraformaldehyde for $8 \mathrm{~h}$ at room temperature, and then embedded in paraffin. Subsequently, the heart samples were cut into 5 - $\mu \mathrm{m}$-thick sections, and separately stained with Hematoxylin-Eosin (HE) and Masson's trichrome as previously described[19]. Digital images were obtained by light microscopy (BX51T-PHD-J11, OLYMPUS, Tokyo, Japan). Image analysis was performed by using Image Pro Plus 6.0 software (Media Cybernetics, USA) to quantify the morphological changes and collagen content of the heart tissues. The data of cardiomyocyte cross sectional area (CSA) was collected from 30 representative cardiomyocytes per field and 3 random fields in each slide. The volume fraction of collagen (CVF) was calculated by a formula (CVF \%= average collagen area / area of total field $\times 100$ ).

\section{Transmission electron microscopy (TEM)}

Transmission electron microscopy was performed to detect the cardiac ultrastructure according to previous protocol[20, 21]. LV samples were harvested and fixed in $2.5 \%$ glutaraldehyde overnight at $4{ }^{\circ} \mathrm{C}$, and post-fixed in $1 \%$ osmic acid. After dehydration, the tissues were embedded with Epon 812 and baked at $60{ }^{\circ} \mathrm{C}$ for $36 \mathrm{~h}$. Ultrathin sections $(50 \sim 60 \mathrm{~nm})$ were obtained and stained with $3 \%$ uranyl acetate-lead citrate, and then examined with a transmission electron microscope (H-7650, HITACHI, Japan). The Mitochondrial morphological parameters on each slide were calculated under six random fields, and the numbers of six slides were averaged for one rat. Mitochondrial morphological characteristics were quantified by Image Pro Plus 6.0 software (Media Cybernetics, USA): (1) mitochondrial area; (2) aspect ratio: defined as the ratio between the major and minor axis of the mitochondria; (3) degree of branching: defined as $\left(\mathrm{Pm}^{2}\right) /(4 \mathrm{pAm}),(\mathrm{Pm}$ is the perimeter of the mitochondrial and Am is the area); (4) the number of mitochondrial vacuolization.

\section{Echocardiography}


Echocardiography was used to evaluate the cardiac function in all of the rats after 4-week drugs administration before sacrificed as described[22] . Ultrasound imaging was obtained with a 4 A VisualSonics high-resolution Vevo 2100 system (VisualSonics Inc., Toronto, Canada) with a 16-MHz linear transducer (MS-250). In brief, rats were anesthetized with $2 \%$ isoflurane and placed in supine position on a heated platform (to maintain a body temperature at $37^{\circ} \mathrm{C}$ ) for continuous electrocardiogram monitoring. After the heart rate (HR) was stable, B-mode images were acquired in the parasternal long-axis and short-axis views, and M-mode images were performed in the parasternal short axis at the mid-papillary level. And then apical four-chamber view was performed to acquire the mitral valve flow via a pulsed wave (PW) Doppler method. All the parameters were assessed in 3 consecutive cardiac cycles at least; mean values from 3 measurements were calculated. And all the analyses were performed in a blinded manner.

\section{Conventional echocardiography}

The conventional parameters, including the left ventricular end-diastolic inner dimension (LVIDd), left ventricular end-systolic inner dimension (LVIDs), diastolic left ventricular anterior wall thickness (LVAWd), systolic left ventricular anterior wall thickness (LVAWs), diastolic left ventricular posterior wall thickness (LVPWd), systolic left ventricular posterior wall thickness (LVPWs), left ventricular end-diastolic volume (LVEDV), left ventricular end-systolic volume (LVESV), ejection fraction (EF), fractional shortening (FS), stroke volume (SV) and cardiac output (CO) were obtained and calculated form the M-mode images in the parasternal short axis to assess the systolic function. The early (E) and late (A) peak mitral valve flow velocity was analyzed using PW Doppler imaging in the apical four-chamber view to evaluate the diastolic function.

\section{Cardiac time intervals (CTI)}

Cardiac time intervals were measured by mitral valve flow Doppler imaging combined with an electrocardiogram through apical fourchamber view[23, 24] . Isovolumic relaxation time (IVRT) was defined as the time interval from aortic valve closing to mitral valve opening, which was used for diastolic function evaluation. Isovolumic contraction time (IVCT), indicating systolic function evaluation, was defined as the time interval from mitral valve closure to aortic valve opening. Aortic ejection time (AET) defined as the time interval from aortic valve opening to aortic valve closing. Myocardial performance index (MPI) was calculated as the sum of IVRT and IVCT divided by the AET: (IVRT + IVCT) / AET, which was a parameter of both systolic and diastolic function.

\section{Speckle-tracking echocardiography (STE)}

Speckle-tracking echocardiography was obtained in parasternal long-axis and short-axis views [16, 25]. B-mode images with a frame rate above $200 \mathrm{frames} / \mathrm{s}$ were used, and then the LV endocardium and epicardium were traced manually in end diastole. For the long-axis view global longitudinal strain (GLS) and strain rate (GLRS) was calculated, and for the short-axis view global radial strain (GRS), global radial strain rate (GRRS), global circumferential strain (GCS) and global circumferential strain rate (GCRS) were obtained. Global strain and strain rate values were analyzed from the average of the 6 segments of the LV.

\section{Quantitative real time PCR (qRT-PCR)}

Total mRNA of the LV samples were extracted using TRIzol reagent (Invitrogen, Carlsbad, CA) according to the manufacturer's protocol[26]. Detailed methodology is available in the online-only Data Supplement.

\section{Statistical analysis}

The values are presented as means \pm SD. Unpaired Student's t-tests were used for comparisons between two groups. Differences among three or more groups were analyzed using one-way ANOVA. Receiver operating characteristic (ROC) curves were studied to determine sensitivity and specificity of cardiac functional parameters to detect myocardial damage. Linear regression analysis was performed between continuous variables using Pearson's correlation coefficient (r). Statistical analysis was performed with GraphPad Prism software 8.0 (GraphPad Software Inc., USA) and IBM SPSS Statistics 26.0 (IBM Corporation, USA). $P$ values $<0.05$ were considered as statistically significant.

\section{Results}

\section{The Changes of Cardiac Remodelling by ROCK inhibition}

STZ treated rats showed stable characteristics of diabetes, including significantly decreased BW and increased FBG (Table 1). However, short-term treatment by Fasudil had no effect on BW and FBG (Table 1). Cardiac remodelling is a hallmark of the structure damage in diabetic cardiomyopathy, which is featured with cardiac hypertrophy and fibrosis. HW/BW and HW/TL ratio were significantly increased in 
diabetic rats (HW/BW: CON vs DM, $P=0.001 ; \mathrm{HW} / \mathrm{TL}$ : CON $v s \mathrm{DM}, P=0.022 ;$ Fig.1 \& Table 1 ) and reduced in the DMF group but with no significance (Fig.1). Histological analysis showed that the CSA of myocardial cells increased significantly in the DM group (CON $v s$ DM, $1.72 \times 10^{-3} \pm 2.83 \times 10^{-4}$ vs $3.37 \times 10^{-3} \pm 5.63 \times 10^{-4} \mathrm{~mm}^{2}, P<0.001$; Fig. $1 \mathrm{e}$ ). Meanwhile, myocardial fibrosis was proved by Masson's trichrome staining in diabetic rats (CON vs DM, $0.034 \pm 0.014$ vs $0.126 \pm 0.020 \%, P<0.001$; Fig. 1f). After fasudil treatment, the DMF group revealed remarkable alleviation of cardiac structure damage compared with the DM group. (CSA: DM vs DMF, $3.37 \times 10^{-3} \pm 5.63 \times 10^{-4}$ vs $2.41 \times 10^{-3} \pm$ $1.96 \times 10^{-4} \mathrm{~mm}^{2}, P<0.001$; CVF: DM vs DMF, $0.126 \pm 0.020$ vs $0.087 \pm 0.014 \%, P=0.001$; Fig. 1 ), but not to the normal level.

\section{Evaluation of Cardiac Ultrastructure by Transmission Electron Microscopy}

The mitochondrial morphology characteristics determined by stereology investigations directly reveal ultrastructure changes in cardiomyocytes. Remarkable changes in the morphological appearance of mitochondria were shown in diabetic rats, including the smaller mitochondrial area, the increased aspect ratio and degree of mitochondrial branching, and the higher number of distorted vacuous mitochondria (Fig.2), which indicated the progressive impairments in cardiac ultrastructure. Compared with the DM group, short-term ROCK inhibition significantly restored the mitochondrial area (DM vs DMF, $0.53 \pm 0.05 v s 0.66 \pm 0.09 \mu \mathrm{m}^{2}, P=0.005$; Fig.2b), reduced the mitochondrial aspect ratio (DM vs DMF, $2.48 \pm 0.28 v s 1.83 \pm 0.10, P<0.001$; Fig.2c), reduced the degree of mitochondrial branching (DM $v s$ DMF, $1.81 \pm 0.12$ vs $1.56 \pm 0.07, P=0.001$; Fig.2d) and alleviated the vacuolization in mitochondria (DM vs DMF, $42.67 \pm 5.24$ vs $19.00 \pm$ 10.30, $P<0.001$; Fig.2e) in the DMF group. In addition, ROCK inhibition improved the imbalance of mitochondrial division/fusion by upregulating Mfn1 and Mfn2 mRNA transcriptions and downregulating Drp1 and Fis1 mRNA transcriptions (Fig.S2).

\section{Evaluation of Cardiac Function by Conventional Echocardiography}

The diabetic rats showed a noteworthy decline in HR, from 354 bmp to 295 bmp, and an enlargement in LV characterized by the increased LVIDd and LVEDV, compared with the CON group (Table S2). However, ROCK inhibition had no significant effect on the HR in diabetic rats. The diabetic rats had impaired LV systolic performance evidenced by the reductions of EF and FS, 26\% and $34 \%$ respectively (Fig. 3 \& Table S2). Meanwhile, the CO was significantly depressed in the DM group, however the SV had no change (Fig.3 \& Table S2). In the DMF group, $\mathrm{CO}$ and SV were significantly restored (CO: DM vs DMF, $76.67 \pm 15.64$ vs $95.90 \pm 11.49 \mathrm{ml} / \mathrm{min}, P=0.034 ;$ SV: DM vs DMF, $259 \pm 34$ vs $312 \pm$ $46 \mu \mathrm{l}, P=0.034$; Fig.3), but EF and FS did not show the same trend. In addition, cardiac diastolic dysfunction was evidenced by a reduced mitral valve E/A in the DM group compared to the controls (CON vs DM, $1.53 \pm 0.23$ vs $1.22 \pm 0.10, P=0.043$; Fig.3g). Interestingly, a 4-week treatment by Fasudil failed to improve the mitral valve E/A in diabetic rats (Fig. $3 \mathrm{~g}$ ).

\section{Evaluation of Cardiac Function by Cardiac Time Intervals}

CTI measured from mitral valve flow Doppler imaging were combined with an electrocardiogram to assess the alterations of cardiac function in all of the rats. IVCT, IVRT and MPI were prolonged in diabetic rats (IVCT: CON vs DM, $19.72 \pm 1.75$ vs $30.96 \pm 7.21 \mathrm{~ms}, P=0.008$; IVRT: CON vs DM, $19.97 \pm 2.81$ vs $31.63 \pm 3.68 \mathrm{~ms}, P<0.001$; MPI: CON vs DM, $0.63 \pm 0.03$ vs $0.93 \pm 0.12$, $P<0.001$; Fig.4), indicating a delayed filling rates at the transmittal flow profile. Notably, ROCK inhibition obviously ameliorated IVCT and MPI (IVCT: DM vs DMF, $30.96 \pm 7.21$ vs $22.05 \pm 6.39 \mathrm{~ms}, P=0.029$; MPI: DM vs DMF, $0.93 \pm 0.12$ vs $0.79 \pm 0.06, P=0.037$; Fig.4) in the DMF group, but none of other variables reached statistical significance.

\section{Evaluation of Myocardial Deformation by Speckle-Tracking Echocardiography}

Myocardial strain parameters were measured by highly sensitive STE which had been demonstrated as a reliable approach to detect myocardial deformation and cardiac impairments (Fig.5 \& Table S3). Compared with the CON group, GLS and GLSR were markedly decreased in diabetic rats, reflecting the damage of myocardial deformation in the longitudinal direction (GLS: CON vs DM, $P<0.001$; GLSR: CON vs DM, $P<0.001$; Fig.5c \& d). These impairments were prevented in the DMF group, whereby GLS exhibited a markedly increase but GLSR showed no significance (GLS: DM vs DMF, $P<0.001$; Fig.5c). A similar pattern was found in the circumferential direction where GCS and GCSR were reduced in diabetic rats (GCS: CON vs DM, $P<0.001$; GCSR: CON $v s \mathrm{DM}, \mathrm{P}<0.001$; Fig.5e \& f), meanwhile, both of them were significantly ameliorated in the DMF group (GCS: DM vs DMF, $P=0.003$; GCSR: DM $v s$ DMF, $P=0.021$; Fig.5e \& f). In the radial direction, though GRS and GRSR were reduced in diabetic rats, only the strain rate revealed a significant change (CON $v s$ DM, $P=0.024$, Fig.5h). However, ROCK inhibition had no significant effect on both GRS and GRSR in the DMF group (Fig.5g \& h). Additionally, FAC was attenuated in the diabetic cardiomyopathy animals (CON vs DM, $P<0.001$, Fig.5i) and subsequently increased in the DMF group (DM vs DMF, $P<0.001$, Fig.5i).

\section{Diagnostic Assessment of Myocardial Damage}


Figure 6 summarized the relative cardiac function alterations induced by diabetes and intervention compared with the control group by a spider's web plot. Healthy controls were shown as a blue circle, which was normalized to 1 . Changes in the DM group were marked by the red circle and in the DMF group were marked by the green one. Similar deviations were observed in the treated and untreated diabetic rats. Noteworthily, treatment in the DMF group tried to drive the green circle to the healthy shape via influencing several functional parameters and structural parameters (Fig.6).

To assess the validity of the parameters as the predictors of diabetic cardiomyopathy, ROC curves were displayed in Table 2. EF, FS and MV E/A had superior sensitivity and specificity for diabetic cardiomyopathy among the conventional parameters, at a cutoff value of $65.62 \%$, $35.07 \%$ and 1.25 , respectively (Fig.7a \& Table 2). Similarly, the significant area under the ROC curves and the prominent sensitivity and specificity in the CTI parameters, including IVCT, IVRT and MPI, also made them possible to distinguish subtle myocardial damages induced by diabetes (Fig.7b). From this analysis, the cutoff values of IVCT, IVRT and MPI were proposed at $23.33 \mathrm{~ms}, 25.56 \mathrm{~ms}$, and 0.71 (Table 2) respectively. Among STE parameters, FAC, GCS and GCSR were the superior predictors for the detection of myocardial damage at a cutoff value of $48 \%,-17.14 \%$ and $-4.031 / \mathrm{s}$, respectively (Fig.7c \& Table 2). GLSR and GRSR also allowed to differentiate the damage and nondamage cardiomyocytes induced by diabetes (Table 2). Other STE parameters, GLS and GRS failed to distinguish the myocardial damage due to the lack of the significance in the ROC curves analysis. Notably, the ability to predict myocardial damage were comparable among the CTI parameters (MPI, IVRT) and the STE parameter (GLSR), which all indicated the cardiac diastolic function (Table 3). Furthermore, the parameters characterized the cardiac systolic function, including MPI, IVCT, FAC, GCR, GCSR and GRSR were demonstrated the nearly equivalent validity according to our study (Table 3 ).

Linear regression analysis was performed to clarify the associations between echocardiographic parameters and cardiac structural parameters (Table S4). MPI and FAC were strongly associated with the diabetes-induced cardiac hypertrophy and fibrosis (Fig.8 \& Table S4). GCS and GCSR were relevant intensively to cardiac fibrosis but moderately to the CSA of cardiomyocytes (Fig. 8 \& Table S4). The conventional parameters (EF and FS), the CTI parameters (IVCT and IVRT) and the STE parameters (GLS, GLSR and GRSR) all showed the moderate correlations with cardiac hypertrophy and fibrosis respectively (Table S4). Besides, CO and MV E/A showed the low correlations with both two cardiac structural parameters, and SV and GRS showed no significant relationships according to the analysis (Table S4).

\section{Discussion}

The major findings in our study were that: ROCK inhibition improved diabetes-induced myocardial damage both in microstructure and ultrastructure. The early alterations of cardiac dysfunction accompanied by structure changes could be detected both by CTI and STE parameters in diabetic hearts. According to our data, parameters derived from STE were more sensitive to identify the early changes of myocardial damages and quantify the subtle cardiac dysfunction. Moreover, FAC, GCS and GCSR were demonstrated to be noninvasively potential predictors for diabetic cardiomyopathy diagnosis, and valuable and feasible measures to evaluate the therapeutic effect.

Diabetic cardiomyopathy causes severe myocardial damage resulting in the abnormalities of cardiac function and structure. Some diabetic animal models have been described for the study of DM and its complications, predominantly in rodents[3, 27]. In our study, we used STZhigh-fat diet T2DM rat models combined with a longer amount of time to mimic the common structural and functional phenotype of the diabetic human hearts[3,28]. Experimental and clinical researches have shown that cardiac hypertrophy and fibrosis both contribute to LV remodeling[2, 28]. In line with previous studies, we found the significant augmentation of myocardial hypertrophy and fibrosis in diabetic rat heart, which indicated that STZ-induced diabetic rats in our study developed into cardiac remodeling under the long-term influence of diabetic state.

Numerous molecular signaling pathways have involved in the pathophysiological process of diabetic cardiomyopathy[2, 3, 28]. Recent studies revealed the significant role of RhoA/ROCK signaling pathway in the underlying mechanisms of diabetic cardiomyopathy[5, 6]. The Rho-associated coiled-coil containing kinases (ROCKs) are members of the AGC serine/threonine kinase family, and are downstream effectors of RhoA, a member of small GTP-binding protein. There are two isoforms of ROCKs, known as ROCK1 and ROCK2, encoded by two different genes[29]. Activated ROCK contributes to the cardiomyocyte apoptosis, cardiac hypertrophy and fibrosis[5, 30, 31], and mediates vascular smooth muscle cell contraction and several proinflammatory, thrombogenic and fibrogenic molecules[5]. Notably, recent study showed that T2DM patients treated with glucose-lowering drugs, antihypertensive treatment and statins were of significant increase in ROCK activation, suggesting the additional role of ROCK inhibition in the prevention of T2DM[32]. These highlight the importance of RhoA/ROCK signaling pathway in diabetic heart therapy. Moreover, our previous studies have shown ROCK inhibition improved the cardiac structure abnormalities in diabetic hearts[8-10]. In the present study, we used Fasudil, a ROCK inhibitor[33], to inhibit RhoA/ ROCK signaling pathway in diabetic animal models. After 4-week treatment, ROCK inhibition prominently alleviated cardiac hypertrophy and fibrosis, suggesting a cardioprotective effects against cardiac remodeling in diabetic rats. The cardiac ultrastructure in diabetic rats was confirmed by TEM detection, which characterized by dumbbell-shaped mitochondria for disturbed fusion, smaller fragment and even the distorted vacuous 
mitochondria for exacerbated fission. Mitochondrial dysfunction and impaired energetics characterize the diabetic heart ultrastructural damage $[2,4,28,34]$. Previous studies have proved that RhoA/Rho kinase pathway is involved in the regulation of the mitochondrial dynamics in some cases[35-38]. In our study, ROCK inhibition improved the disturbed mitochondrial fission/fusion in diabetic rats and facilitated mitochondrial fusion and uncoupling to restore the cardiac ultrastructural abnormalities. These indicated that ROCK inhibition improved the abnormalities both in cardiac microstructure and ultrastructure.

Accompanied by the changes in structure, it is the cardiac dysfunction. Correspondingly, we found the impaired LV systolic and diastolic performance in diabetic rats. Our animal models shared numerous features with diabetic cardiomyopathy including the enlarged LV, remarkable myocardial hypertrophy and fibrosis and obvious dysfunction. Interestingly, ROCK inhibition increased the SV and CO to a certain degree, possibly relevant to an integrated consequence of the slightly elevated LVEDV and HR. However, the classical parameters, EF, FS and MV E/A, derived from the conventional echocardiography were almost not changed in the presence of 4-week treatment with fasudil. Notably, Lai[6] have observed significant improvements of cardiac dysfunction in diabetic rats treated with fasudil for 8 weeks via modulation of $\mathrm{Ca}^{2+}$ handling and actin remodeling. That suggesting that the duration of administration might be a key factor for the different results, and the traditional detection methods of the cardiac function in the early stage of treatment might be another. Accordingly, EF, FS and MV E/A, the conventional parameters, might be insensitive to the early and subtle alterations in cardiac dysfunction along with the improvements in structure in diabetic rats.

Because of the results in our study and others have demonstrated the limitations of EF, FS and MVE/A in evaluating the early alterations of cardiac dysfunction[17, 39], we used CTI and STE to characterize the diabetic myocardial damage and assess the therapeutic effect respectively. CTI parameters defined by the opening and closing of the heart valves have been suggested and investigated in kinds of cardiovascular disease[23, 40]. Previous studies have shown that IVCT was prolonged and AET was shortened in HF[23, 41, 42]. In addition, AET has been proved to be a predictor of the outcome in primary pulmonary hypertension and ischemic heart disease[42]. Furthermore, MPI is demonstrated a superior marker of cardiovascular morbidity and mortality than conventional parameters[43]. In diabetic populations or animals, MPI has been found increased with a prolonged IVCT and IVRT and a shortened AET [43-47]. Similar findings were obtained in our study, where the diabetic rats had significantly increased IVCT, IVRT and MPI. Noticeably, the diabetic rats with worsen LV systolic and diastolic dysfunction were improved by inhibiting RhoA/ROCK pathway. The early and subtle changes of cardiac structure could be detected by IVCT and MPI. CTI parameters, in particular the IVCT and MPI, are feasible in the early detection of cardiac function alterations and might be predictive of the therapeutic effect in diabetic cardiomyopathy.

Further, the present study showed the benefits of STE in predicting diagnosis and therapy in diabetic cardiomyopathy. Myocardial deformation measured by STE is broadly governed by myocardial microstructure. The LV longitudinal mechanics are predominantly determined by subendocardium, while the circumferential and radial mechanics are decided by the mid-wall and the subepicardium[48, 49]. Myocardial strain is well known to account for ventricular vascular coupling, as decreased myocardial strain is known to attenuate myocardial efficiency[50]. Damage in different myocardial layers leads to distinct phenotypes in cardiac dysfunction[48], which make it possible to classify heart disease according to layer-specific alterations. Damage mainly in subendocardium may be accompanied by diastolic dysfunction but preserved EF together with reduced longitudinal but unaltered radial and circumferential mechanics in patients and animals[15,51]. Thus, GLS is well-accepted marker of subendocardial damage and linked to subendocardial fibrosis[52]. For another, an acute transmural insult involving in the subepicardial and midmyocardial dysfunction results in a reduction in LV circumferential, radial and twist mechanics as well as a decrease in EF[48]. Consistent with this theory, Niu[14] fount the reduced longitudinal and radial mechanics in pressure overload-induced adult rats along with a severely impaired contractile function, which was featured with the LV stiffness in the whole layers[14]. In diabetic humans and animal models, the LV function is obviously impaired with reduced myocardial strain and strain rate[17, 53]. This is in accordance with our findings, where global strain and strain rate were markedly decreased in diabetic rats in the longitudinal, circumferential and radial directions, suggesting a transmural cardiac remodeling. Inhibiting RhoA/ROCK signaling pathway significantly ameliorated GLS, GCS and GCSR, which manifested the improvements of the cardiac hypertrophy and fibrosis in the global heart. Additionally, mitochondria are the powerhouse of the cell, which continuously provide large amounts of adenosine triphosphate (ATP) to cardiomyocytes to maintain cardiac function[34]. The improvements of cardiac ultrastructure also contributed to restore the global strain and strain rate in the DMF group. Moreover, FAC, reflecting the degree of thickening in the radial direction[48], was attenuated in diabetic rats and subsequently increased by ROCK inhibition. Accordingly, it might be suggested that the changes of cardiac microstructure and ultrastructure could be detected by STE parameters at the early stage of the treatment compared with conventional parameters. STE parameters could be suitable markers for predicting diagnosis and therapeutic effect and providing the quantitative assessment of layerspecific cardiac function alterations.

The present study has demonstrated that ROCK inhibition improved diabetes-induced myocardial hypertrophy and fibrosis, and the alterations of cardiac dysfunction accompanied by structure changes were detected both by CTI and STE parameters (Figure 6). However, what kind of parameters is the optimal diagnostic approach to assess the subtle cardiac damage and predict the therapeutic effect in the

Page $7 / 21$ 
early stage of diabetic cardiomyopathy remains to reveal. Hence, we demonstrated the validity of parameters as predictors for diabetic cardiomyopathy by ROC curves and linear regression analyses. Numerous studies have been identified the sensitivity and specificity of conventional parameters for cardiovascular disease diagnosis[3, 4, 12].Similar findings were confirmed in our study. Whereas, the mild ability of indicating diabetic myocardial hypertrophy and fibrosis made the conventional parameters fail to detect the subtle alterations of cardiac function. CTI parameters, such as IVCT, IVRT, MPI, showed prominent sensitivity and specificity of cardiac systolic and diastolic function respectively, which are consistent with previous data[43]. For the strong correlations with cardiac microstructure, MPI was a best index to distinguish diabetes-induced myocardial damage, compared with IVCT and IVRT. As previously reported, MPI is a superior predictor of cardiovascular morbidity and mortality in the general population, and can provide prognostic information of cardiovascular risk factors[40].The findings in our study also confirmed that MPI offers the therapeutic information on the early alterations both of cardiac systolic and diastolic dysfunction.

Parameters derived from STE, including FAC, GCS and GCS, were demonstrated superior predictors for the detection of diabetes-induced myocardial damage in our study. According to analysis, FAC, GCS and GCSR were sensitive and specific for detecting the early and subtle alterations in cardiac dysfunction. Previous literature has shown that GLS parameters are detectable in corresponding microstructural and functional changes in subendocardium, and are associated with the subendocardial fibrosis[15]. However, in our study GLS and GLSR just exhibited a moderate relationship with cardiac fibrosis and hypertrophy in the whole fiber layers, which made it a lower significance in differentiating damage and non-damage in cardiomyocytes in comparison with the other STE parameters. Nevertheless, GLS was obviously enhanced in the DMF group, suggesting the probable improvements in subendocardium and the high potential to predict the cardiac diastolic function alterations in the early stage of treatment. We further compared the area under the ROC curve for distinguishing the most suitable diagnostic markers for diabetic cardiomyopathy. Interestingly, CTI parameters (MPI and IVRT) and STE parameters (GLS, FAC, GCS and GCSR) shared the equal capacity for the diagnosis of diabetic cardiomyopathy. Moreover, CTI parameters were mainly exhibited the specificity in predicting the cardiac dysfunction, but STE parameters the sensitivity. As the understanding of layer-specific structure changes to the myocardial deformation helps in estimating the transmural disease burden correctly, and provides pathophysiologic insight into the mechanisms of LV dysfunction[48]. The advantage that STE parameters could provide the additional information on the layer-specific pathologies in diabetic heart made it superior to CTI parameter.

\section{Limitations}

Several possible limitations should be considered. Firstly, the drug for diabetes treatment is a major limitation in the present study. Though RhoA/ROCK signaling pathway is of increasing importance in diabetic heart therapy, ROCK inhibition is not the classic way for diabetic cardiomyopathy therapy compared with glucose control by Metformin. However, STE is valuable and feasible in detection of the early and subtle cardiac functional alterations, which still has provided new insight into diabetic cardiomyopathy management. Further we will improve our animal models to challenge more useful methods for the diagnosis of diabetic cardiomyopathy and provide more preclinical data for translational medicine. Secondly, the current number of the investigated rats may serve as a limitation. Nevertheless, the sample size is based on statistical calculations according to previous observations, in addition to the reasonability in experimental studies. So, the sample size should be expanded to solve this problem, which must be a part of future translational studies. Finally, despite that we discussed the ability of STE parameters for indicating the damage in different fiber layers on the basis of prior studies, we did not correlate the functional parameters with structural parameters, including myocardial hypertrophy and fibrosis, in different myocardial fiber layers respectively. Even so, that does not exert a significant impact on the way of drawing to the conclusion based on our data in scientific manners.

\section{Conclusion}

In conclusion, our data suggesting that the early and subtle alterations of cardiac function in diabetic hearts are detectable by CTI and STE parameters simultaneously. The comparison between CTI and STE parameters has identified STE strain and strain rate to be preferable diagnostic measures for the early detection and quantification of layer-specific functional alterations. Furthermore, FAC, GCS and GCSR are proved to be potential predictive indexes of cardiac dysfunction in diabetic cardiomyopathy. Our study provides translational evidence for a better understanding on the pathophysiologic progress of diabetic cardiomyopathy during management.

\section{Abbreviations}

DM: Diabetes mellitus; T2DM: type 2 diabetes mellitus; HF: heart failure; CTI: cardiac time intervals; STE: speckle-tracking echocardiography; HFD: high-fat diet; STZ: streptozocin; TEM: transmission electron microscopy; ROC: receiver operating characteristic; BW: body weight; FBG: fasting blood glucose; HR: heart rate; HW: heart weight; HW/BW: heart weight / bodyweight; HW/TL: heart weight / tibia length; CSA: cross sectional area; CVF: collagen volume fraction; EF: ejection fraction; FS: fractional shortening; CO: cardiac output; SV: stroke volume; MV E/A: 
mitral valve E/A; LVPWd: diastolic left ventricular posterior wall thickness; LVPWs: systolic left ventricular posterior wall thickness; LVIDd: left ventricular end-diastolic inner dimension; LVIDs: left ventricular end-systolic inner dimension; LVEDV: Left ventricular end-diastolic volume; LVESV: left ventricular end-systolic volume; AET: aortic ejection time; IVCT: isovolumic contraction time; IVRT: isovolumic relaxation time; MPI: myocardial performance index; GLS: global longitudinal strain; GLSR: global longitudinal strain rate; GCS: global circumferential strain; GCSR: global circumferential strain rate; FAC: fractional area change; GRS: global radial strain; GRSR: global radial strain rate.

\section{Declarations}

\section{Authors' contributions}

CZY designed the study, researched data and drafted the manuscript, QYC contributed to preparation, editing and review of the manuscript, WLJ, MMF, LN, and LZ performed the experiments, LSY and XLX provided oversight for the project and participated in editing of the manuscript, GBY and LYJ is the guarantor of this work.

\section{Funding}

This study was supported by National Natural Science Foundation of China $(81570345,81400217)$, Natural Science Foundation of Hebei Province (H2020206504, H2017206358)

\section{Acknowledgements}

We appreciate Shuguang Ren and his members (the Experimental Animal Center, the Fourth Hospital of Hebei Medical University) for their help in performing animal models, and Huaxing Zhang (the Central Laboratory, Hebei Medical University) for his excellent technical assistance.

\section{Ethics approval and consent to participate}

The studies were approved by the research ethic committee of Hebei Medical University (Shijiazhuang, China), in accordance with the Helsinki Declaration.

\section{Availability of data and materials}

All data generated or analyzed during this study are included in this published article.

\section{Consent for publication}

Not applicable.

\section{Competing interests}

The authors declare that they have no competing interests.

\section{References}

1. Cho NH, Shaw JE, Karuranga S, Huang Y, da Rocha Fernandes JD, Ohlrogge AW, Malanda B. IDF Diabetes Atlas: Global estimates of diabetes prevalence for 2017 and projections for 2045. Diabetes Res Clin Pract. 2018;138:271-281.

2. Gopal K, Chahade JJ, Kim R, Ussher JR. The Impact of Antidiabetic Therapies on Diastolic Dysfunction and Diabetic Cardiomyopathy. Front Physiol. 2020;11:603247.

3. Ritchie RH, Abel ED. Basic Mechanisms of Diabetic Heart Disease. Circ Res. 2020;126(11):1501-1525.

4. Peterson LR, Gropler RJ. Metabolic and Molecular Imaging of the Diabetic Cardiomyopathy. Circ Res. 2020;126(11):1628-1645.

5. Dai Y, Luo W, Chang J. Rho kinase signaling and cardiac physiology. Curr Opin Physiol. 2018;1:14-20.

6. Lai D, Gao J, Bi X, He H, Shi X, Weng S, Chen Y, Yang Y, Ye Y, Fu G. The Rho kinase inhibitor, fasudil, ameliorates diabetes-induced cardiac dysfunction by improving calcium clearance and actin remodeling. J Mol Med (Berl). 2017;95(2):155-165.

7. Katare R, Pearson JT, Lew JK, Wei M, Tsuchimouchi H, Du CK, Zhan DY, Umetani K, Shirai M, Schwenke DO. Progressive Decrease in Coronary Vascular Function Associated With Type 2 Diabetic Heart Disease. Front Physiol. 2018;9:696.

8. Zhou H, Zhang KX, Li YJ, Guo BY, Wang M, Wang M. Fasudil hydrochloride hydrate, a Rho-kinase inhibitor, suppresses high glucoseinduced proliferation and collagen synthesis in rat cardiac fibroblasts. Clin Exp Pharmacol Physiol. 2011;38(6):387-394.

Page $9 / 21$ 
9. Yang R, Chang L, Liu S, Jin X, Li Y. High glucose induces Rho/ROCK-dependent visfatin and type I procollagen expression in rat primary cardiac fibroblasts. Mol Med Rep. 2014;10(4):1992-1998.

10. Zhou H, Sun Y, Zhang L, Kang W, Li N, Li Y. The RhoA/ROCK pathway mediates high glucose-induced cardiomyocyte apoptosis via oxidative stress, JNK, and p38MAPK pathways. Diabetes/Metabolism Research and Reviews. 2018;34(6).

11. Grilo GA, Shaver PR, Stoffel HJ, Morrow CA, Johnson OT, Iyer RP, de Castro Bras LE. Age- and sex-dependent differences in extracellular matrix metabolism associate with cardiac functional and structural changes. J Mol Cell Cardiol. 2020;139:62-74.

12. Scherrer-Crosbie M, Thibault HB. Echocardiography in translational research: of mice and men. J Am Soc Echocardiogr. 2008;21(10):1083-1092.

13. Terminology, Diagnostic Criteria Committee of The Japan Society of Ultrasonics in M. Standard measurement of cardiac function indexes. J Med Ultrason (2001). 2006;33(2):123-127.

14. Niu P, Li L, Yin Z, Du J, Tan W, Huo Y. Speckle tracking echocardiography could detect the difference of pressure overload-induced myocardial remodelling between young and adult rats. J R Soc Interface. 2020;17(163):20190808.

15. Beyhoff N, Lohr D, Foryst-Ludwig A, Klopfleisch R, Brix S, Grune J, Thiele A, Erfinanda L, Tabuchi A, Kuebler WM et al. Characterization of Myocardial Microstructure and Function in an Experimental Model of Isolated Subendocardial Damage. Hypertension. 2019;74(2):295304.

16. Kraker K, O'Driscoll JM, Schutte T, Herse F, Patey O, Golic M, Geisberger S, Verlohren S, Birukov A, Heuser A et al. Statins Reverse Postpartum Cardiovascular Dysfunction in a Rat Model of Preeclampsia. Hypertension. 2020;75(1):202-210.

17. Matyas C, Kovacs A, Nemeth BT, Olah A, Braun S, Tokodi M, Barta BA, Benke K, Ruppert M, Lakatos BK et al. Comparison of speckletracking echocardiography with invasive hemodynamics for the detection of characteristic cardiac dysfunction in type-1 and type-2 diabetic rat models. Cardiovasc Diabetol. 2018;17(1):13.

18. Bulani Y, Sharma SS. Argatroban Attenuates Diabetic Cardiomyopathy in Rats by Reducing Fibrosis, Inflammation, Apoptosis, and Protease-Activated Receptor Expression. Cardiovasc Drugs Ther. 2017;31(3):255-267.

19. Yang F, Qin Y, Wang Y, Meng S, Xian H, Che H, Lv J, Li Y, Yu Y, Bai Y et al. Metformin Inhibits the NLRP3 Inflammasome via AMPK/mTORdependent Effects in Diabetic Cardiomyopathy. Int J Biol Sci. 2019;15(5):1010-1019.

20. Mollica MP, Mattace Raso G, Cavaliere G, Trinchese G, De Filippo C, Aceto S, Prisco M, Pirozzi C, Di Guida F, Lama A et al. Butyrate Regulates Liver Mitochondrial Function, Efficiency, and Dynamics in Insulin-Resistant Obese Mice. Diabetes. 2017;66(5):1405-1418.

21. Hsieh CC, Li CY, Hsu CH, Chen HL, Chen YH, Liu YP, Liu YR, Kuo HF, Liu PL. Mitochondrial protection by simvastatin against angiotensin II-mediated heart failure. Br J Pharmacol. 2019;176(19):3791-3804.

22. Chen Z, Yu H, Shi Y, Zhu M, Wang Y, Hu X, Zhang Y, Chang Y, Xu M, Gao W. Vascular Remodelling Relates to an Elevated Oscillatory Shear Index and Relative Residence Time in Spontaneously Hypertensive Rats. Sci Rep. 2017;7(1):2007.

23. Alhakak AS, Sengelov M, Jorgensen PG, Bruun NE, Johnsen C, Abildgaard U, Iversen AZ, Hansen TF, Teerlink JR, Malik FI et al. Left ventricular systolic ejection time is an independent predictor of all-cause mortality in heart failure with reduced ejection fraction. Eur $\mathrm{J}$ Heart Fail. 2020.

24. Hotta K, Chen B, Behnke BJ, Ghosh P, Stabley JN, Bramy JA, Sepulveda JL, Delp MD, Muller-Delp JM. Exercise training reverses ageinduced diastolic dysfunction and restores coronary microvascular function. J Physiol. 2017;595(12):3703-3719.

25. Chowdhury SAK, Warren CM, Simon JN, Ryba DM, Batra A, Varga P, Kranias EG, Tardiff JC, Solaro RJ, Wolska BM. Modifications of Sarcoplasmic Reticulum Function Prevent Progression of Sarcomere-Linked Hypertrophic Cardiomyopathy Despite a Persistent Increase in Myofilament Calcium Response. Front Physiol. 2020;11:107.

26. Xiao H, Li H, Wang JJ, Zhang JS, Shen J, An XB, Zhang CC, Wu JM, Song Y, Wang XY et al. IL-18 cleavage triggers cardiac inflammation and fibrosis upon beta-adrenergic insult. Eur Heart J. 2018;39(1):60-69.

27. Riehle C, Bauersachs J. Of mice and men: models and mechanisms of diabetic cardiomyopathy. Basic Res Cardiol. 2018;114(1):2.

28. Parim B, Sathibabu Uddandrao VV, Saravanan G. Diabetic cardiomyopathy: molecular mechanisms, detrimental effects of conventional treatment, and beneficial effects of natural therapy. Heart Fail Rev. 2019;24(2):279-299.

29. Nakagawa O, Fujisawa K, Ishizaki T,Saito Y, Nakao K, Narumiya S. ROCK-I and ROCK-II, two isoforms of Rho-associated coiled-coil forming protein serine/threonine kinase in mice. FEBS Lett. 1996;392(2):189-93.

30. Santos GL, Hartmann S, Zimmermann WH, Ridley A, Lutz S. Inhibition of Rho-associated kinases suppresses cardiac myofibroblast function in engineered connective and heart muscle tissues. J Mol Cell Cardiol. 2019;134:13-28.

31. Ocaranza MP, Moya J, Jalil JE, Lavandero S, Kalergis AM, Molina C, Gabrielli L, Godoy I, Cordova S, Castro P et al. Rho-kinase pathway activation and apoptosis in circulating leucocytes in patients with heart failure with reduced ejection fraction. $J$ Cell Mol Med.

2020;24(2):1413-1427.

Page 10/21 
32. Ocaranza MP, Valderas P, Moya J, Gabrielli L, Godoy I, Cordova S, Nab PM, Garcia L, Farias L, Jalil JE. Rho kinase cascade activation in circulating leukocytes in patients with diabetes mellitus type 2. Cardiovasc Diabetol. 2020;19(1):56.

33. Suda A, Takahashi J, Hao K, Kikuchi Y, Shindo T, Ikeda S, Sato K, Sugisawa J, Matsumoto Y, Miyata S et al. Coronary Functional Abnormalities in Patients With Angina and Nonobstructive Coronary Artery Disease. J Am Coll Cardiol. 2019;74(19):2350-2360.

34. Gollmer J, Zirlik A, Bugger H. Mitochondrial Mechanisms in Diabetic Cardiomyopathy. Diabetes Metab J. 2020;44(1):33-53.

35. Preau S, Delguste F, Yu Y, Remy-Jouet I, Richard V, Saulnier F, Boulanger E, Neviere R. Endotoxemia Engages the RhoA Kinase Pathway to Impair Cardiac Function By Altering Cytoskeleton, Mitochondrial Fission, and Autophagy. Antioxid Redox Signal. 2016;24(10):529-542.

36. Shen YL, Shi YZ, Chen GG, Wang LL, Zheng MZ, Jin HF, Chen YY. TNF-a induces Drp1-mediated mitochondrial fragmentation during inflammatory cardiomyocyte injury. Int J Mol Med. 2018;41(4):2317-2327.

37. Liu Z, Li H, Su J, Xu S, Zhu F, Ai J, Hu Z, Zhou M, Tian J, Su Z et al. Numb Depletion Promotes Drp1-Mediated Mitochondrial Fission and Exacerbates Mitochondrial Fragmentation and Dysfunction in Acute Kidney Injury. Antioxid Redox Signal. 2019;30(15):1797-1816.

38. Wang W, Wang Y, Long J, Wang J, Haudek SB, Overbeek P, Chang BH, Schumacker PT, Danesh FR. Mitochondrial fission triggered by hyperglycemia is mediated by ROCK1 activation in podocytes and endothelial cells. Cell Metab. 2012;15(2):186-200.

39. Hoffman M, Kyriazis ID, Lucchese AM, de Lucia C, Piedepalumbo M, Bauer M, Schulze PC, Bonios MJ, Koch WJ, Drosatos K. Myocardial Strain and Cardiac Output are Preferable Measurements for Cardiac Dysfunction and Can Predict Mortality in Septic Mice. J Am Heart Assoc. 2019;8(10):e012260.

40. Dons M, Olsen FJ, de Knegt MC, Fritz-Hansen T, Mogelvang R, Alhakak AS, Jespersen T, Gislason G, Biering-Sorensen T. Myocardial performance index by tissue Doppler echocardiography predicts adverse events in patients with atrial fibrillation. Eur Heart $J$ Cardiovasc Imaging. 2020;21(5):560-566.

41. Bruch C, Schmermund A, Marin D, Katz M, Bartel T, Schaar J, Erbel R. Tei-index in patients with mild-to-moderate congestive heart failure. Eur Heart J. 2000;21(22):1888-1895.

42. Sztrymf B, Günther S, Artaud-Macari E, Savale L, Jaïs X, Sitbon O, Simonneau G, Humbert M, Chemla D. Left ventricular ejection time in acute heart failure complicating precapillary pulmonary hypertension. Chest. 2013;144(5):1512-1520.

43. Biering-Sorensen T, Jensen JS, Andersen HU, Rossing P, Jensen MT. Cardiac time intervals and the association with 2D-speckle-tracking, tissue Doppler and conventional echocardiography: the Thousand\&1 Study. Int J Cardiovasc Imaging. 2016;32(5):789-798.

44. Kranstuber AL, Del Rio C, Biesiadecki BJ, Hamlin RL, Ottobre J, Gyorke S, Lacombe VA. Advanced glycation end product cross-link breaker attenuates diabetes-induced cardiac dysfunction by improving sarcoplasmic reticulum calcium handling. Front Physiol. 2012;3:292.

45. Sanches IC, Buzin M, Conti FF, Dias DDS, Dos Santos CP, Sirvente R, Salemi VMC, Llesuy S, Irigoyen MC, De Angelis K. Combined aerobic and resistance exercise training attenuates cardiac dysfunctions in a model of diabetes and menopause. PLoS One.

2018;13(9):e0202731.

46. Sanhal CY, Daglar HK, Kara O, Uygur D, Yucel A. Assessment of fetal myocardial performance index in women with pregestational and gestational diabetes mellitus. J Obstet Gynaecol Res. 2017;43(1):65-72.

47. Goroshi M, Chand D. Myocardial Performance Index (Tei Index): A simple tool to identify cardiac dysfunction in patients with diabetes mellitus. Indian Heart J. 2016;68(1):83-87.

48. Mor-Avi V, Lang RM, Badano LP, Belohlavek M, Cardim NM, Derumeaux G, Galderisi M, Marwick T, Nagueh SF, Sengupta PP et al. Current and evolving echocardiographic techniques for the quantitative evaluation of cardiac mechanics: ASE/EAE consensus statement on methodology and indications endorsed by the Japanese Society of Echocardiography. J Am Soc Echocardiogr. 2011;24(3):277-313.

49. Kraker K, Schutte T, O'Driscoll J, Birukov A, Patey O, Herse F, Muller DN, Thilaganathan B, Haase N, Dechend R. Speckle Tracking Echocardiography: New Ways of Translational Approaches in Preeclampsia to Detect Cardiovascular Dysfunction. Int J Mol Sci. 2020;21(3).

50. Brar PC, Chun A, Fan X, Jani V, Craft M, Bhatla P, Kutty S. Impaired myocardial deformation and ventricular vascular coupling in obese adolescents with dysglycemia. Cardiovasc Diabetol. 2019;18(1):172.

51. Ikonomidis I, Tzortzis S, Triantafyllidi H, Parissis J, Papadopoulos C, Venetsanou K, Trivilou P, Paraskevaidis I, Lekakis J. Association of impaired left ventricular twisting-untwisting with vascular dysfunction, neurohumoral activation and impaired exercise capacity in hypertensive heart disease. Eur J Heart Fail. 2015;17(12):1240-1251.

52. Beyhoff N, Brix S, Betz IR, Klopfleisch R, Foryst-Ludwig A, Krannich A, Stawowy P, Knebel F, Grune J, Kintscher U. Application of SpeckleTracking Echocardiography in an Experimental Model of Isolated Subendocardial Damage. J Am Soc Echocardiogr. 2017;30(12):12391250 e1232. 
53. Jedrzejewska I, Krol W, Swiatowiec A, Wilczewska A, Grzywanowska-Laniewska I, Dluzniewski M, Braksator W. Left and right ventricular systolic function impairment in type 1 diabetic young adults assessed by 2D speckle tracking echocardiography. Eur Heart $J$ Cardiovasc Imaging. 2016;17(4):438-446.

\section{Tables}

Table.1 Basic Characteristics of the diabetic rats.

\begin{tabular}{|c|c|c|c|c|c|c|c|c|c|c|c|c|}
\hline & $\mathrm{CON}$ & & & DM & & & DMF & & & CONF & & \\
\hline $\mathrm{FBG}(\mathrm{mmol} / \mathrm{l})$ & 6.07 & \pm & 0.48 & 16.95 & \pm & $1.76^{\star \star \star}$ & 17.84 & \pm & $3.20^{\star \star \star}$ & 5.78 & \pm & 0.55 \\
\hline BW (g) & 689 & \pm & 82 & 446 & \pm & $151^{\star \star \star}$ & 469 & \pm & $52^{\star \star \star}$ & 656 & \pm & 37 \\
\hline HW (g) & 1.85 & \pm & 0.14 & 2.00 & \pm & 0.36 & 1.97 & \pm & 0.20 & 1.85 & \pm & 0.17 \\
\hline $\operatorname{LVW}(\mathrm{g})$ & 1.32 & \pm & 0.19 & 1.44 & \pm & 0.28 & 1.42 & \pm & 0.16 & 1.31 & \pm & 0.14 \\
\hline $\mathrm{TL}(\mathrm{cm})$ & 4.81 & \pm & 0.09 & 4.44 & \pm & $0.17^{\star \star \star}$ & 4.59 & \pm & $0.08^{\star \star \#}$ & 4.71 & \pm & 0.04 \\
\hline $\mathrm{HW} / \mathrm{BW}$ & 0.0027 & \pm & 0.0005 & 0.0049 & \pm & $0.0016^{\star *}$ & 0.0043 & \pm & $0.0008^{*}$ & 0.0029 & \pm & 0.0003 \\
\hline $\mathrm{HW} / \mathrm{TL}$ & 0.38 & \pm & 0.02 & 0.45 & \pm & $0.07^{*}$ & 0.43 & \pm & 0.04 & 0.39 & \pm & 0.04 \\
\hline LVW/HW & 0.71 & \pm & 0.06 & 0.72 & \pm & 0.02 & 0.72 & \pm & 0.04 & 0.71 & \pm & 0.02 \\
\hline
\end{tabular}

Values are presented as the mean $\pm \mathrm{SD} .{ }^{*} P<0.05,{ }^{*} P<0.01,{ }^{*} * * P<0.001$ vs CON group; ${ }^{\#} P<0.05,{ }^{\# \#} P<0.001,{ }^{\# \# \#} P<0.001$ vs DM group. CON: control rats, DM: diabetic rats, DMF: diabetic rats and fasudil, CONF: control rats and fasudil. FBG: fasting blood glucose, BW: body weight, HW: heart weight, LVW: left ventricle weight, TL: tibia length, HW/BW: heart weight / body weight, HW/TL: heart weight / tibia length, LVW/HW: left ventricle weight / heart weight.

Table 2. Receiver operating characteristics. 


\begin{tabular}{|c|c|c|c|c|c|c|c|c|c|c|c|c|c|}
\hline \multirow{2}{*}{$\begin{array}{l}\text { Parameter } \\
\text { EF }\end{array}$} & \multicolumn{2}{|c|}{$\begin{array}{l}\text { AUC } \\
(95 \% \mathrm{Cl})\end{array}$} & \multirow{2}{*}{$\begin{array}{l}\text { Z } \\
\text { statistic } \\
20.39\end{array}$} & \multirow{2}{*}{$\begin{array}{l}P \\
\text { value } \\
<0.001\end{array}$} & \multirow{2}{*}{$\begin{array}{l}\text { Optimal } \\
\text { cut off } \\
65.62(\%)\end{array}$} & \multicolumn{4}{|c|}{$\begin{array}{l}\text { Sensitivity } \\
(95 \% \mathrm{Cl})\end{array}$} & \multicolumn{4}{|c|}{$\begin{array}{l}\text { Specificity } \\
(95 \% \mathrm{Cl})\end{array}$} \\
\hline & 0.979 & $\begin{array}{l}(0.821 \\
\text { to } \\
1.00)\end{array}$ & & & & 100.00 & ( & $73.5-100.0$ & ) & 91.67 & ( & $61.5-99.8$ & ) \\
\hline FS & 0.979 & $\begin{array}{l}(0.821 \\
\text { to } \\
1.00)\end{array}$ & 22.66 & $<0.001$ & $35.07(\%)$ & 83.33 & ( & $51.6-97.9$ & ) & 100.00 & ( & $73.5-100.0$ & ) \\
\hline CO & 0.639 & $\begin{array}{l}(0.419 \\
\text { to } \\
0.823)\end{array}$ & 1.17 & 0.243 & $89.75(\mathrm{ml} / \mathrm{min})$ & 75.00 & ( & $42.8-94.5$ & ) & 58.33 & ( & $27.7-84.8$ & ) \\
\hline sV & 0.549 & $\begin{array}{l}(0.334 \\
\text { to } \\
0.750)\end{array}$ & 0.39 & 0.695 & $247.47(\mu \mathrm{l})$ & 83.33 & ( & $51.6-97.9$ & ) & 41.67 & ( & $15.2-72.3$ & ) \\
\hline MV E/A & 0.847 & $\begin{array}{l}(0.643 \\
\text { to } \\
0.960)\end{array}$ & 4.24 & $<0.001$ & 1.25 & 83.33 & ( & $51.6-97.9$ & ) & 83.33 & ( & $51.6-97.9$ & ) \\
\hline AET & 0.545 & $\begin{array}{l}(0.331 \\
\text { to } \\
0.747)\end{array}$ & 0.37 & 0.714 & $56.22(\mathrm{~ms})$ & 16.67 & ( & $2.1-48.4$ & ） & 100.00 & ( & $73.5-100.0$ & ) \\
\hline IVCT & 0.736 & $\begin{array}{l}(0.518 \\
\text { to } \\
0.893)\end{array}$ & 2.06 & 0.039 & 23.33(ms) & 66.67 & ( & $34.9-90.1$ & ) & 91.67 & ( & $61.5-99.8$ & ) \\
\hline IVRT & 0.910 & $\begin{array}{l}(0.721 \\
\text { to } \\
0.987)\end{array}$ & 5.29 & $<0.001$ & $25.56(\mathrm{~ms})$ & 91.67 & ( & $61.5-99.8$ & ) & 91.67 & ( & $61.5-99.8$ & ) \\
\hline MPI & 0.931 & $\begin{array}{l}(0.749 \\
\text { to } \\
0.994)\end{array}$ & 6.14 & $<0.001$ & 0.71 & 100.00 & ( & $73.5-100.0$ & ) & 91.67 & ( & $61.5-99.8$ & ) \\
\hline FAC & 0.927 & $\begin{array}{l}(0.744 \\
\text { to } \\
0.993)\end{array}$ & 6.40 & $<0.001$ & $48(\%)$ & 83.33 & ( & $51.6-97.9$ & ) & 100.00 & ( & 73.5 - 100.0 & ) \\
\hline GLS & 0.681 & $\begin{array}{l}(0.461 \\
\text { to } \\
0.854)\end{array}$ & 1.48 & 0.138 & $-10.83(\%)$ & 58.33 & ( & $27.7-84.8$ & ) & 91.67 & ( & $61.5-99.8$ & ) \\
\hline GLSR & 0.799 & $\begin{array}{l}(0.586 \\
\text { to } \\
0.933)\end{array}$ & 3.18 & 0.002 & $-3.22(1 / \mathrm{s})$ & 100.00 & ( & $73.5-100.0$ & ) & 58.33 & ( & $27.7-84.8$ & ) \\
\hline GCS & 0.819 & $\begin{array}{l}(0.610 \\
\text { to } \\
0.945)\end{array}$ & 3.53 & $<0.001$ & $-17.14(\%)$ & 91.67 & ( & $61.5-99.8$ & ) & 66.67 & ( & $34.9-90.1$ & ) \\
\hline GCSR & 0.899 & $\begin{array}{l}(0.707 \\
\text { to } \\
0.984)\end{array}$ & 6.34 & $<0.001$ & $-4.03(1 / \mathrm{s})$ & 100.00 & ( & $73.5-100.0$ & ） & 66.67 & ( & $34.9-90.1$ & ) \\
\hline GRS & 0.528 & $\begin{array}{l}(0.316 \\
\text { to } \\
0.733)\end{array}$ & 0.22 & 0.828 & $27.66(\%)$ & 33.33 & ( & $9.9-65.1$ & ) & 91.67 & ( & $61.5-99.8$ & ) \\
\hline GRSR & 0.740 & $\begin{array}{l}(0.522 \\
\text { to } \\
0.895)\end{array}$ & 2.30 & 0.022 & $4.93(1 / \mathrm{s})$ & 83.33 & ( & $51.6-97.9$ & ) & 58.33 & ( & $27.7-84.8$ & ) \\
\hline
\end{tabular}

AUC: area under the ROC curve, Cl: confidence interval, EF: ejection fraction; FS, fractional shortening, CO: cardiac output, SV: stroke volume, MV E/A: mitral valve E/A, AET: aortic ejection time, IVCT: isovolumic contraction time, IVRT: isovolumic relaxation time, MPI: myocardial performance index, FAC: fractional area change, GLS: global longitudinal strain, GLSR: global longitudinal strain rate, GCS: global circumferential strain, GCSR: global circumferential strain rate, GRS: global radial strain, GRSR: global radial strain rate.

Table 3. Comparisons of the ROC curves. 


\begin{tabular}{|llllllll|}
\hline Variable & $\begin{array}{l}\text { Difference } \\
\text { between area }\end{array}$ & $\begin{array}{l}\text { Standard } \\
\text { Error }\end{array}$ & $\mathbf{9 5 \%} \mathbf{C l}$ & & Z statistic & Pvalue \\
\hline MPI vs GLSR & 0.132 & 0.126 & -0.114 & to & 0.378 & 1.051 & $\mathbf{0 . 2 9 4}$ \\
\hline IVRT vs GLSR & 0.111 & 0.118 & -0.120 & to & 0.342 & 0.944 & $\mathbf{0 . 3 4 5}$ \\
\hline MPI vs FAC & 0.003 & 0.095 & -0.183 & to & 0.190 & 0.037 & $\mathbf{0 . 9 7 1}$ \\
\hline MPI vs GCS & 0.111 & 0.123 & -0.129 & to & 0.351 & 0.907 & $\mathbf{0 . 3 6 5}$ \\
\hline MPI vs GCSR & 0.031 & 0.094 & -0.153 & to & 0.216 & 0.332 & $\mathbf{0 . 7 4 0}$ \\
\hline MPI vs GRSR & 0.191 & 0.107 & -0.020 & to & 0.402 & 1.777 & $\mathbf{0 . 0 7 6}$ \\
\hline IVCT vs FAC & 0.191 & 0.141 & -0.086 & to & 0.468 & 1.353 & $\mathbf{0 . 1 7 6}$ \\
\hline IVCT vs GCS & 0.083 & 0.164 & -0.238 & to & 0.405 & 0.508 & $\mathbf{0 . 6 1 2}$ \\
\hline IVCT vs GCSR & 0.163 & 0.144 & -0.119 & to & 0.446 & 1.132 & $\mathbf{0 . 2 5 8}$ \\
\hline IVCT vs GRSR & 0.003 & 0.136 & -0.264 & to & 0.271 & 0.025 & $\mathbf{0 . 9 8 0}$ \\
\hline
\end{tabular}

IVRT: isovolumic relaxation time, MPI: myocardial performance index, IVCT: isovolumic contraction time, FAC: fractional area change, GLSR: global longitudinal strain rate, GCS: global circumferential strain, GCSR: global circumferential strain rate, GRSR: global radial strain rate.

\section{Figures}



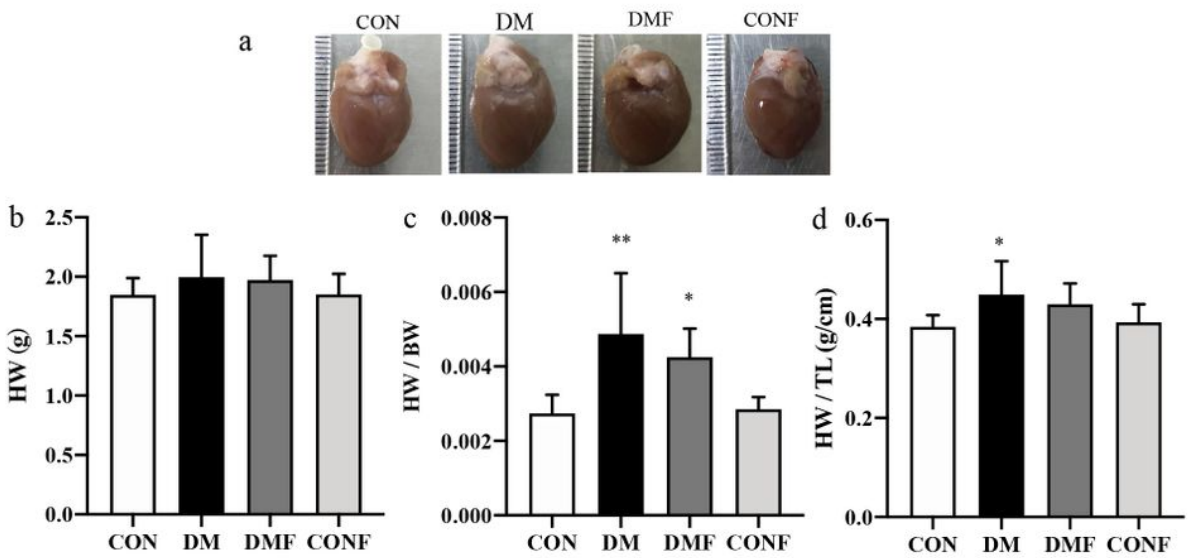

e
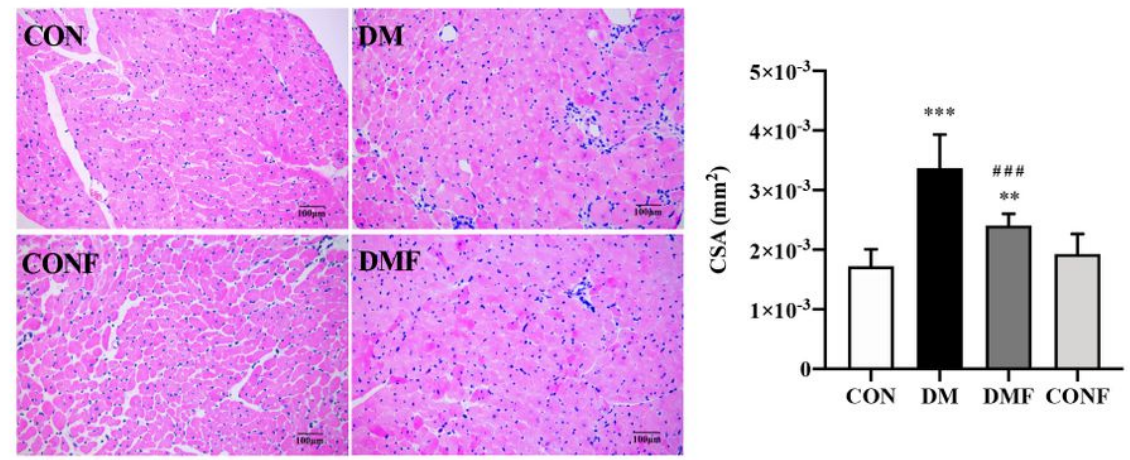

f
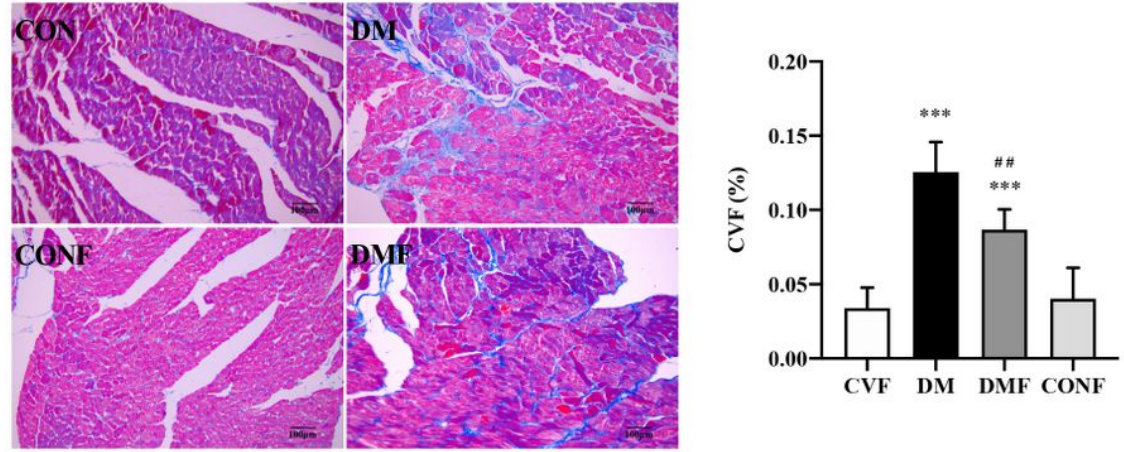

Figure 1

The changes of cardiac remodeling in rats. (a) The heart tissue specimens of the rats were shown, and (b) HW, (c) HW/BW and (d) HW/TL were calculated. (e) The representative images of cardiac cross section stained with H\&E were obtained (magnification 200x), and the CSA of cardiomyocytes were calculated. Scale bar: $100 \mu \mathrm{m}$. (f) The representative histology of mid-myocardial cross-sections performed with Masson's trichrome staining were acquired (magnification 200x). Scale bar: $100 \mu \mathrm{m}$. Quantification of collagen content were analyzed. Values are presented as the mean $\pm \mathrm{SD}$. ${ }^{\star} \mathrm{P}<0.05$, ${ }^{\star *} \mathrm{P}<0.01$, ${ }^{\star * \star} \mathrm{P}<0.001$ vs CON group; $\# \mathrm{P}<0.05, \# \# \mathrm{P}<0.001, \# \# \# \mathrm{P}<0.001$ vs $\mathrm{DM}$ group. CON: control rats, DM: diabetic rats, DMF: diabetic rats and fasudil, CONF: control rats and fasudil. HW: heart weight, HW/BW: heart weight / body weight, HW/TL: heart weight / tibia length, CSA: cross sectional area, CVF: collagen volume fraction. 

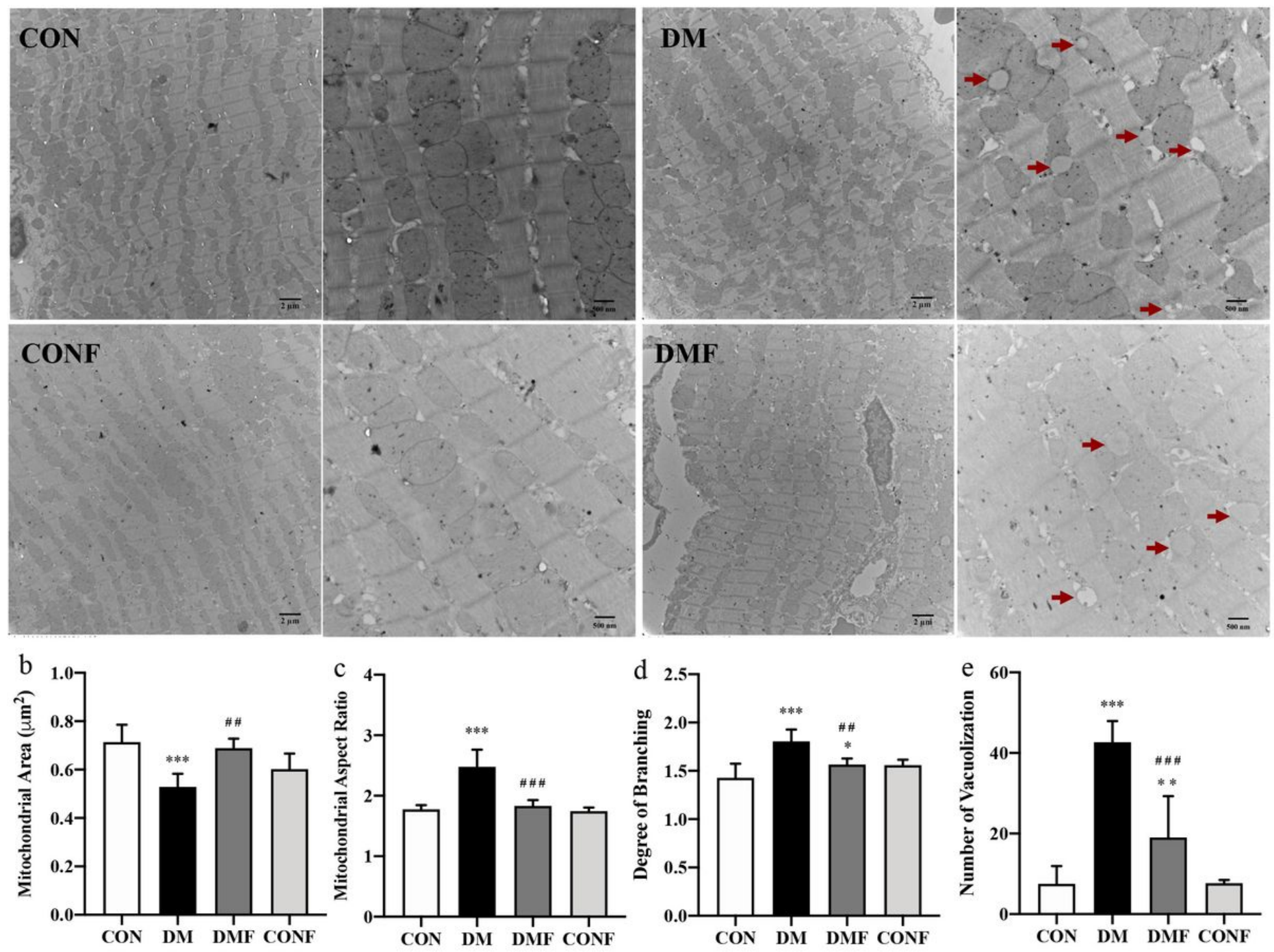

\section{Figure 2}

The cardiac ultrastructure measured by transmission electron microscopy. (a) The representative images of the hearts obtained from transmission electron microscopy were shown. Scale bar: $2 \mu \mathrm{m}$ and $500 \mathrm{~nm}$. Arrows indicated the mitochondria with vacuolization. (b) Mitochondrial area, (c) the aspect ratio, (d) the degree of branching and (e) the number of mitochondria with vacuolization were analyzed.

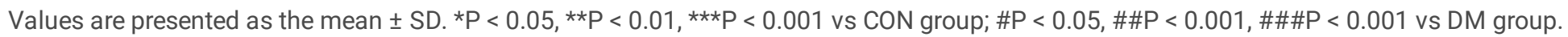
CON: control rats, DM: diabetic rats, DMF: diabetic rats and fasudil, CONF: control rats and fasudil. 
a
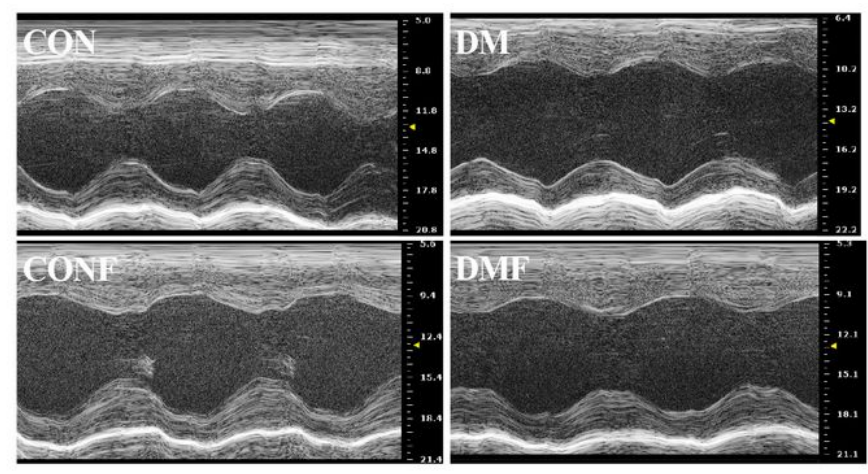

c

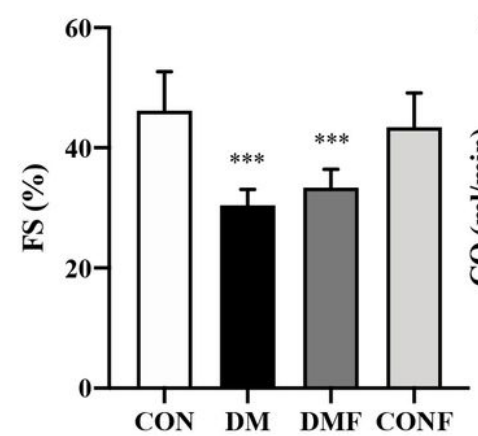

d 150
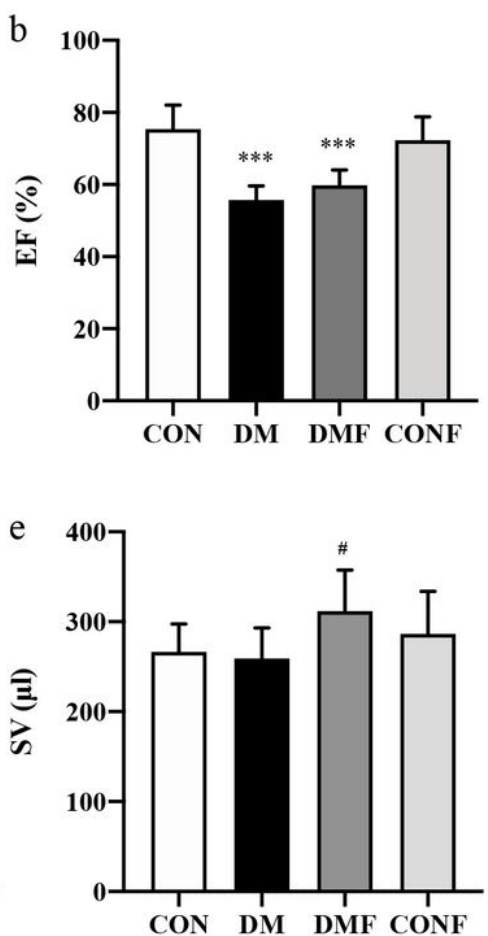

f
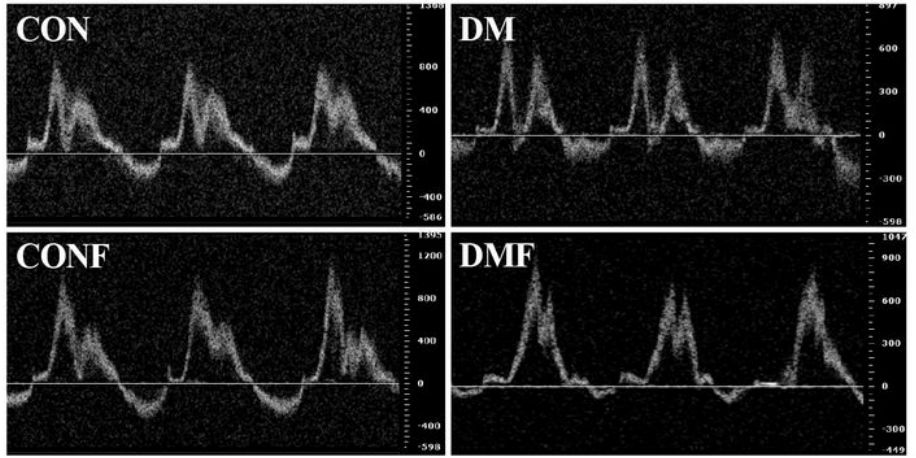

Figure 3

Cardiac dysfunction measured by conventional echocardiography. (a) The M-mode images in parasternal short axis of the rats were acquired, and (b) EF, (c) FS, (d) CO and (e) SV were calculated. (f) The Pulsed wave Doppler of the mitral valve flow was obtained, and (g) peak velocity blood flow in early diastole (the $E$ wave) to peak velocity flow in late diastole (the A wave) ratio was analyzed. Values are

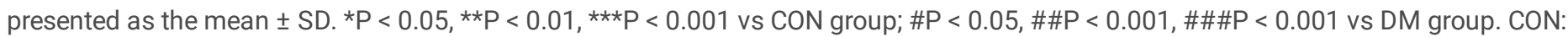
control rats, DM: diabetic rats, DMF: diabetic rats and fasudil, CONF: control rats and fasudil. EF: ejection fraction, FS: fractional shortening, CO: cardiac output, SV: stroke volume, MV E/A: mitral valve E/A. 

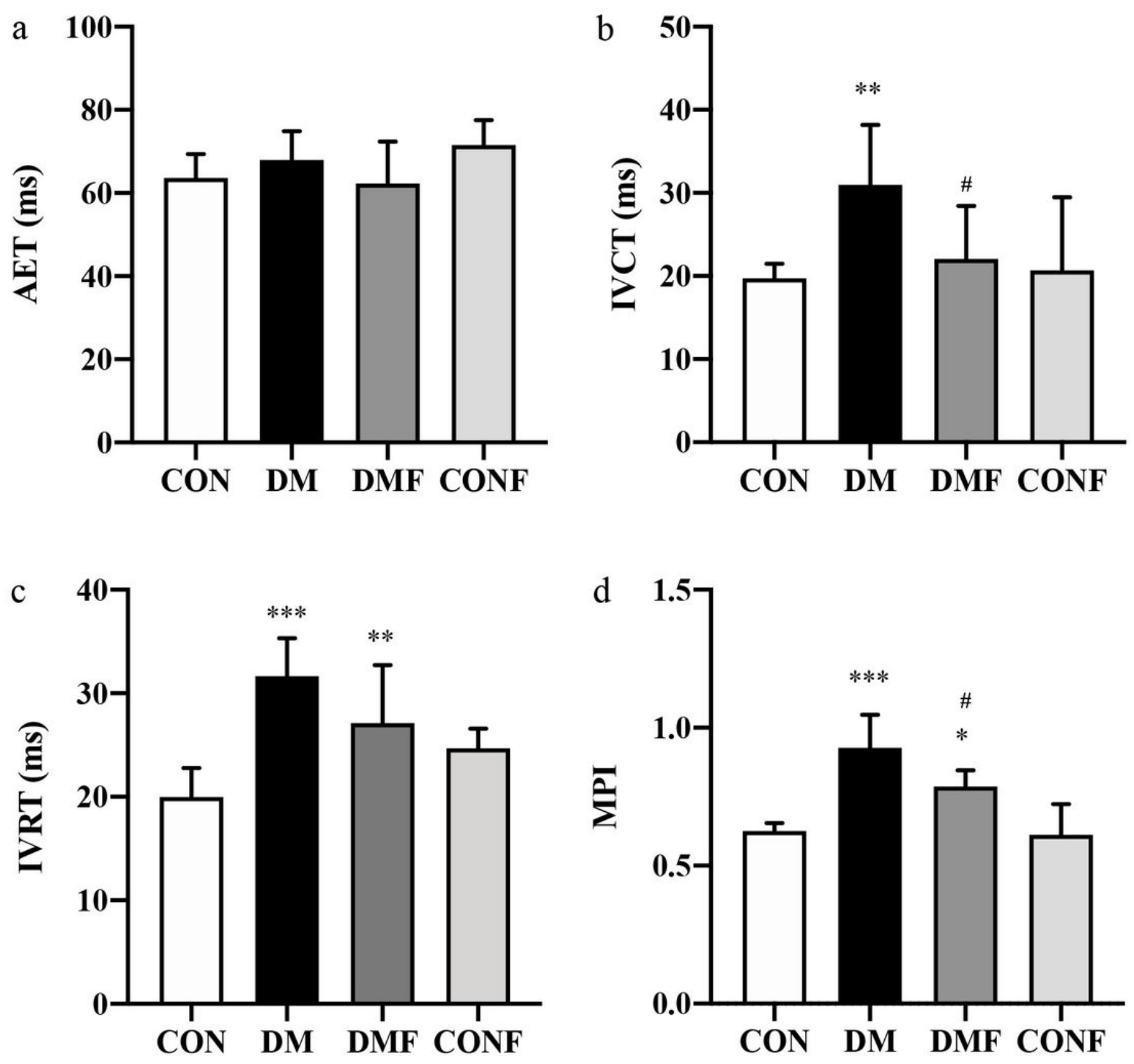

Figure 4

Parameters derived from cardiac time intervals were measured from mitral valve flow Doppler images. (a) AET, (b) IVCT, (c) IVRT, and (d) MPI

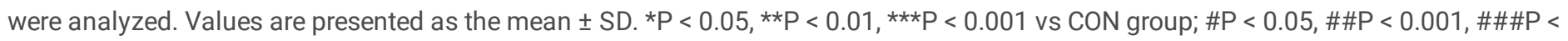
0.001 vs DM group. CON: control rats, DM: diabetic rats, DMF: diabetic rats and fasudil, CONF: control rats and fasudil. AET: aortic ejection time, IVCT: isovolumic contraction time, IVRT: isovolumic relaxation time, MPI: myocardial performance index. 

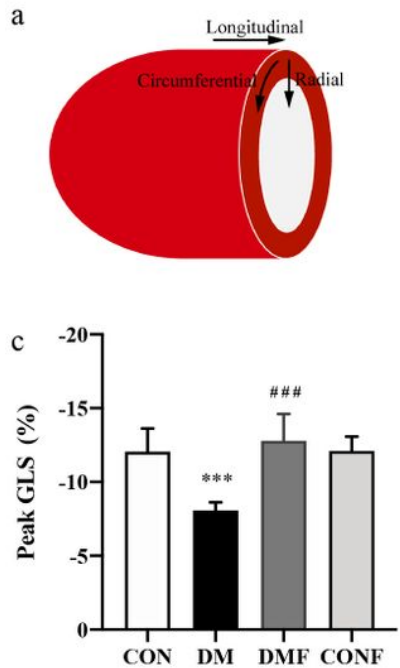

f
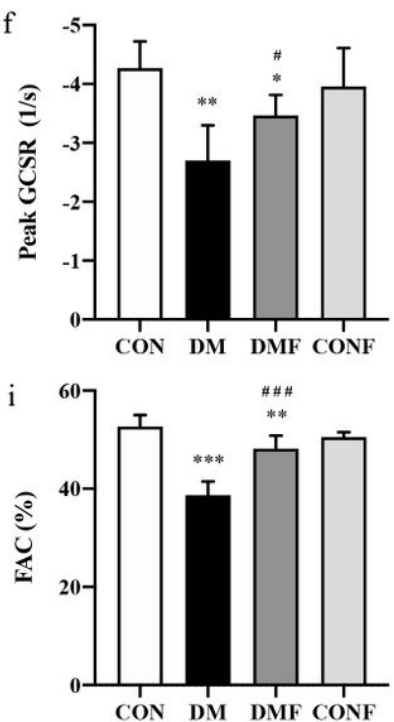

$\mathrm{b}$

CON DM
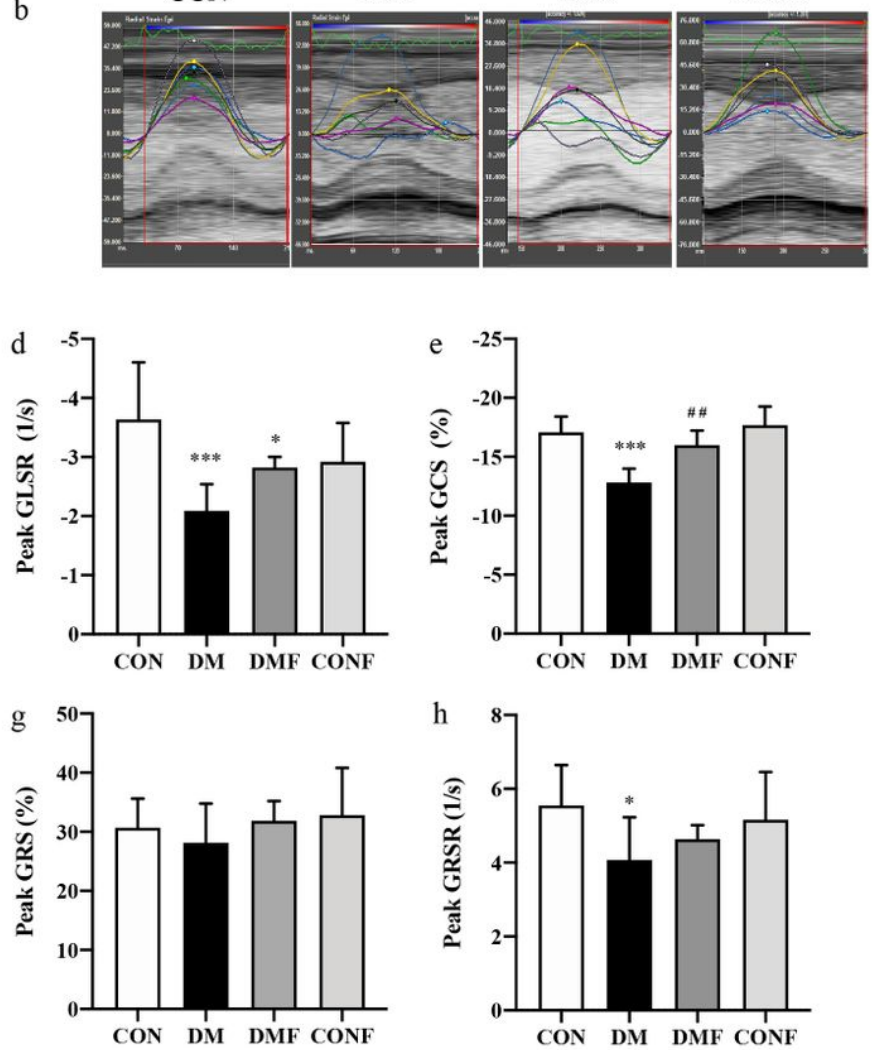

Figure 5

Speckle-tracking echocardiography analysis in rat models. (a) A schematic of the LV with the indication of longitudinal, circumferential and radial strain measurements. (b) The representative longitudinal strain images of STE over one cardiac cycle. The peak values of (c) GLS, (d) GLSR, (e) GCS, (f) GCSR, (g) GRS, (h) GRSR and (i) FAC were calculated. Values are presented as the mean \pm SD. ${ }^{*} \mathrm{P}<0.05$, $* * \mathrm{P}<0.01$, $* \star \star \mathrm{P}<$ 0.001 vs CON group; $\# P<0.05, \# \# P<0.001, \# \# \# P<0.001$ vs DM group. CON: control rats, DM: diabetic rats, DMF: diabetic rats and fasudil, CONF: control rats and fasudil. GLS: global longitudinal strain, GLSR: global longitudinal strain rate, GCS: global circumferential strain, GCSR: global circumferential strain rate, GRS: global radial strain, GRSR: global radial strain rate, FAC: fractional area change. 


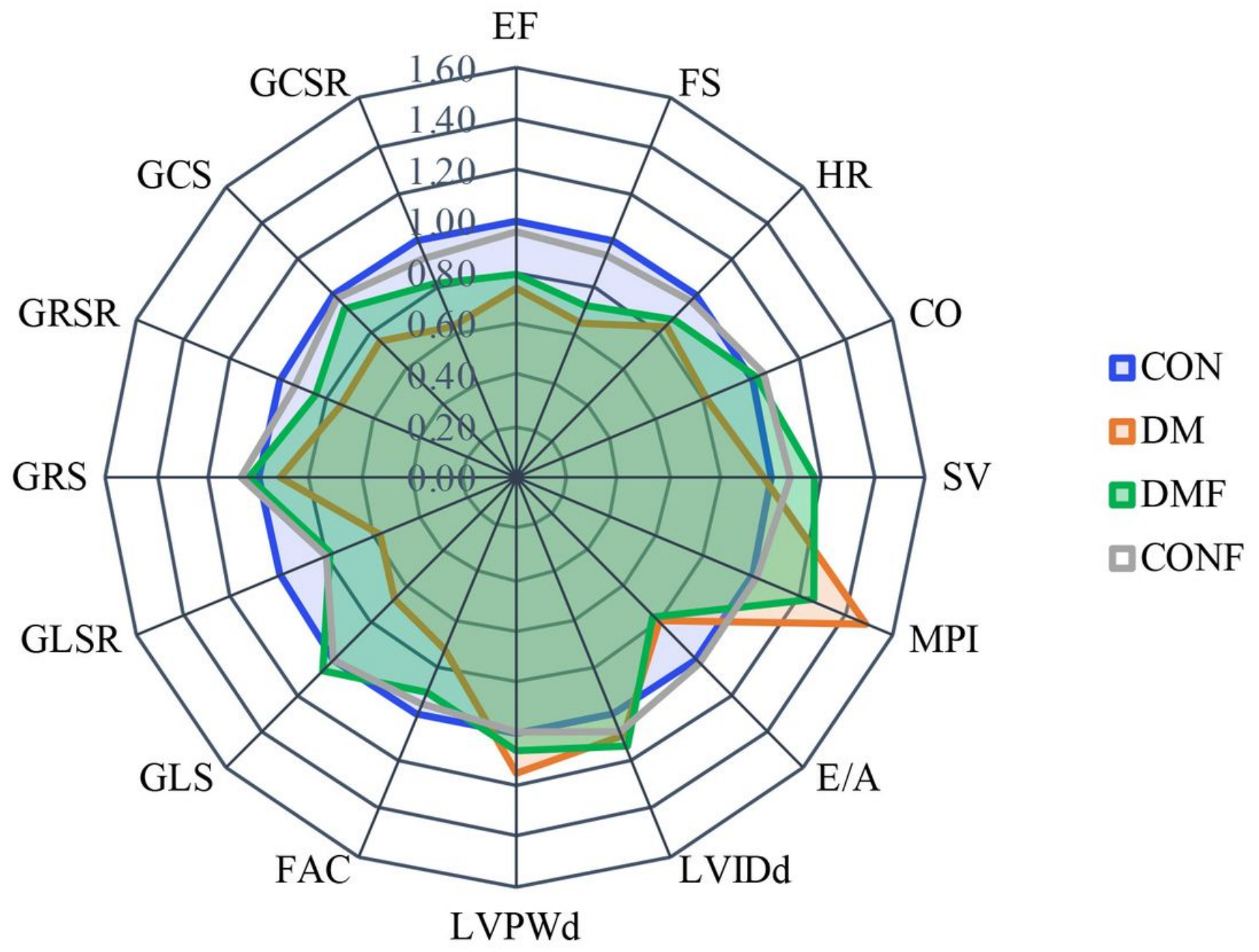

Figure 6

Cardiac function changes. The relative values of the treated and untreated diabetic rats in comparison to the healthy ones were shown. Healthy controls were normalized to one. CON: control rats, DM: diabetic rats, DMF: diabetic rats and fasudil, CONF: control rats and fasudil.
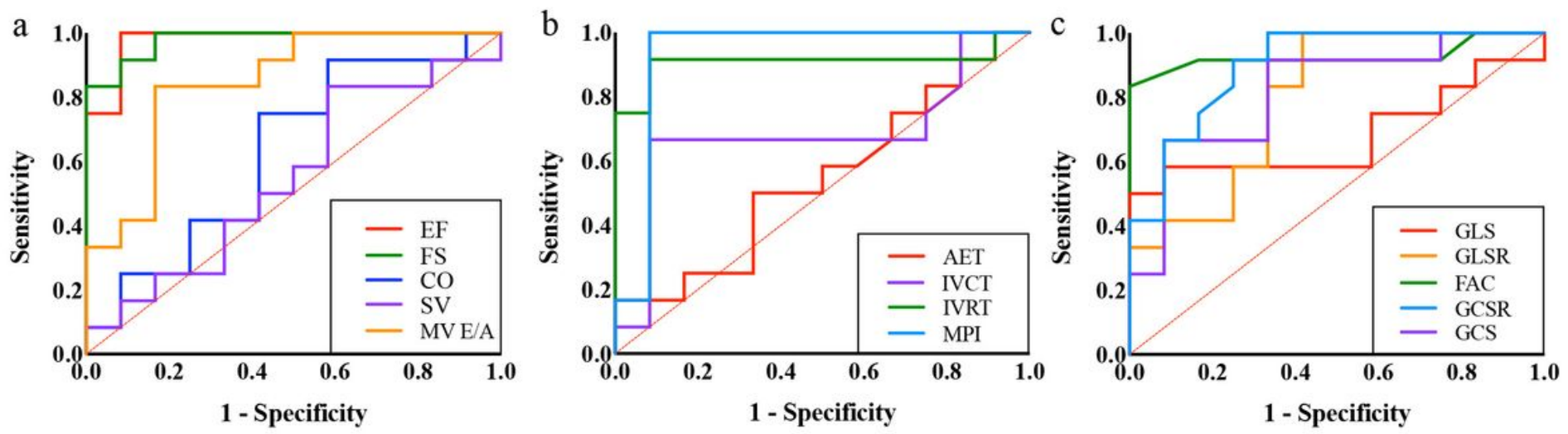

Figure 7

Receiver operating characteristic (ROC) curves for echocardiographic parameters to detect diabetic cardiomyopathy. (a) The ROC curves for conventional parameters, (b) CTI parameters and (c) STE parameters were shown. EF: ejection fraction; FS, fractional shortening, CO: cardiac 
output, SV: stroke volume, MV E/A: mitral valve E/A, AET: aortic ejection time, IVCT: isovolumic contraction time, IVRT: isovolumic relaxation time, MPI: myocardial performance index, GLS: global longitudinal strain, GLSR: global longitudinal strain rate, GCS: global circumferential strain, GCSR: global circumferential strain rate, FAC: fractional area change.

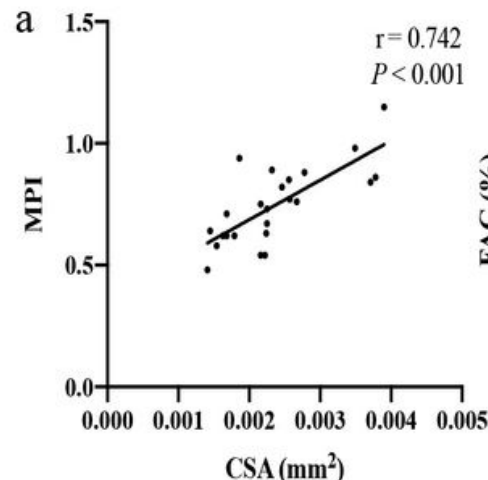

$\operatorname{CSA}\left(\mathrm{mm}^{2}\right)$

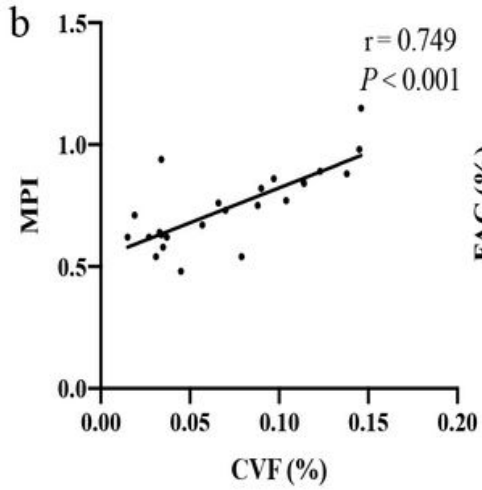

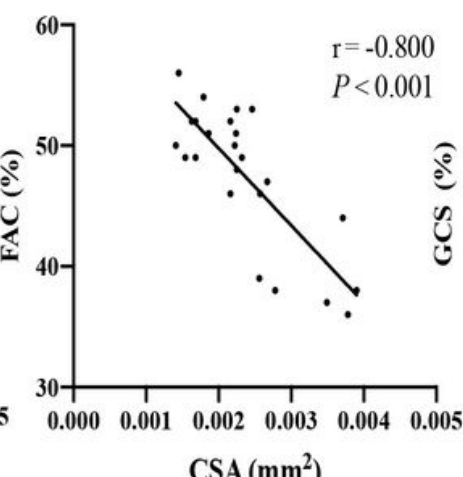

$\operatorname{CSA}\left(\mathrm{mm}^{2}\right)$

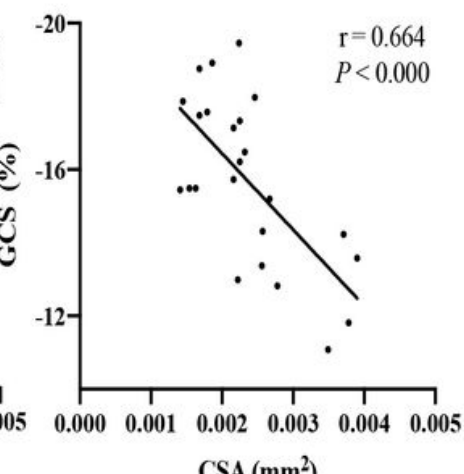

$\operatorname{CSA}\left(\mathrm{mm}^{2}\right)$
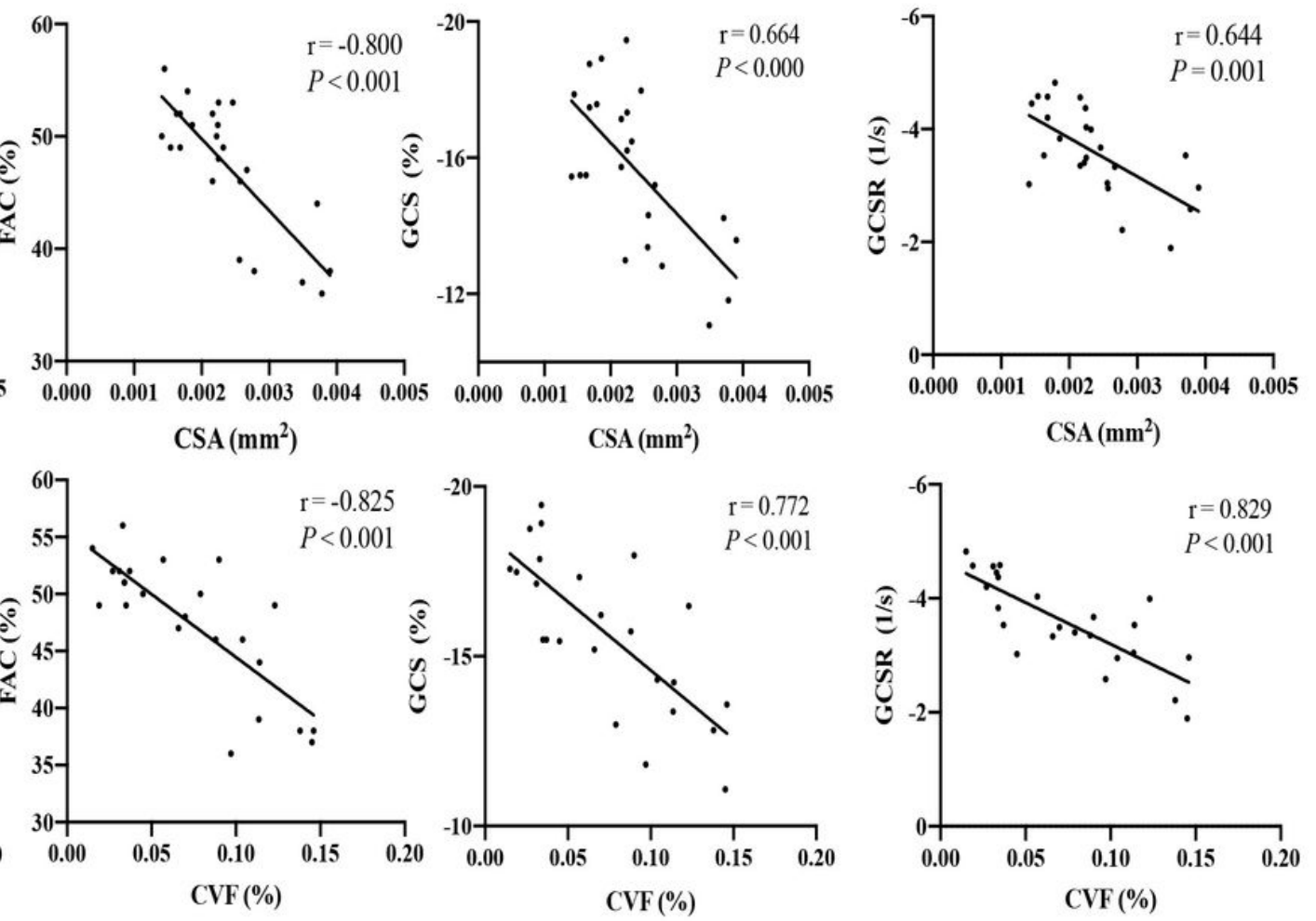

\section{Figure 8}

Correlation analyses. (a) The correlations between functional parameters and CSA of cardiomyocytes. (b) The correlations between functional parameters and cardiac fibrosis. r: linear regression coefficient, MPI: myocardial performance index, FAC: fractional area change, GCS: global circumferential strain, GCSR: global circumferential strain rate, CSA: cross sectional area, CVF: collagen volume fraction.

\section{Supplementary Files}

This is a list of supplementary files associated with this preprint. Click to download.

- LYJSupplementaryMaterials2021327.pdf 